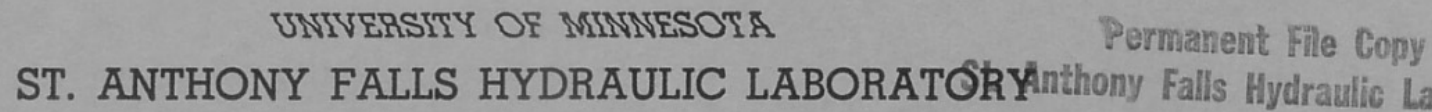

LORENZ G. STRAUB, Director

Technical Paper No. 29, Series B

\title{
Instability of Ventilated Cavities
}

by

E. SILBERMAN and C. S. SONG

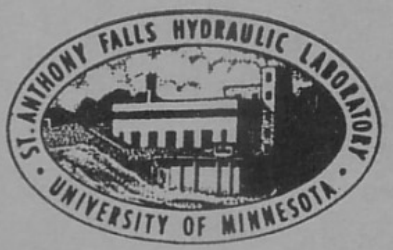

Prepared for

OFFICE OF NAVAL RESEARCH

Department of the Navy

Washington, D. C.

Contract Nonr 710(24), Task NR 062-052

November 1959

Minneapolis, Minnesota 
UNIVERSITY OF MINNESOTA

ST. ANTHONY FALLS HYDRAULIC LABORATORY

LORENZ G. STRAUB, Director

Technical Paper No. 29, Series B

\section{Instability of Ventilated Cavities}

by

E. SILBERMAN and C. S. SONG

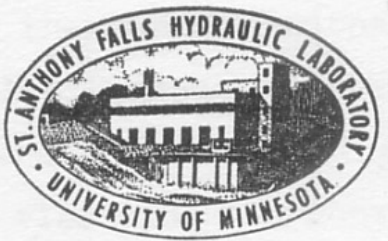

November 1959

Minneapolis, Minnesota 


\section{$\underline{A} \underline{B} \underline{\mathrm{S}} \underline{\mathrm{T}} \underline{\mathrm{R}} \underline{\mathrm{A}} \underline{\mathrm{C}} \underline{\mathrm{T}}$}

Cavitation number $\sigma$ is defined by $\sigma=\left(p_{0}-p_{k}\right) / q$. It can be controlled by controlling the dynamic pressure $q$, the ambient pressure $p_{0}$, or the internal cavity pressure $\mathrm{p}_{\mathrm{k}}$. The present investigation was undertaken to study methods of controlling $\mathrm{p}_{\mathrm{k}}$ by adding air to the wakes of fully submerged bodies. This process has been called ventilation.

It was found that with $p_{0}$ and $q$ fixed, $p_{k}$ increases, and hence $\sigma$ decreases, nearly linearly with the rate of air supply. Numerical values are given in the paper for the normal flat plate, circular cylinder, and various hydrofoils, and these values may be extrapolated to other sizes of bodies than those tested.

It was also found that once $\sigma$ was decreased to a certain critical value, further increase in the air supply rate would not produce a continuing linear decrease in $\sigma$. Rather, $\sigma$ remained nearly constant and the cavities began to vibrate. Vibration occurred with one, two, or more waves on the cavity surface. Only by going from a one-wave to a two-wave cavity could $\sigma$ be reduced further, and the reduction was discontinuous A detailed description of the vibrating cavities is given.

Both two-dimensional and finite aspect-ratio bodies were tested. The tests were conducted in the two-dimensional, vertical, free-jet water tunnel at the St. Anthony Falls Hydraulic Laboratory. All bodies tested, regardless of aspect ratio and whether lifting or nonlifting, behaved quite similarly within both the vibrating and nonvibrating regimes. That the vibration was not peculiar to the vertical free-jet tunnel was demonstrated by comparison with results from a hydrofoil towed in a tank. In the case of lifting bodies of finite span operating near a free surface, air was found to enter, rather than leave, the cavities through the trailing vortexes。 


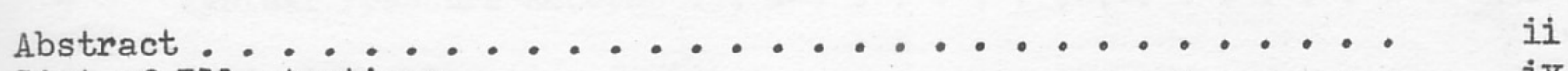

Iist of Illustrations. ..................... iv

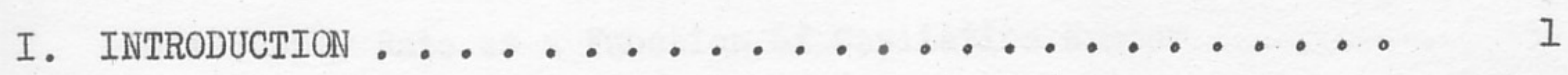

II. EXPERTMENTAL APPARATUS .......................... 2

III. BEHAVIOR OF VENTILATED CAVITIES. . . . . . . . . . .

A. Two-Dimensional Cavities . . . . . . . . . . . . . . . . . . . . . .

C. Boundary Effects ................. 9 9

D. Details of the Vibrating Motion. . . . . . . . 9

IV. EXPERTMENTAL RESULTS ................... 12

A. Air Supply and Cavitation. . . . . . . . . . . 12

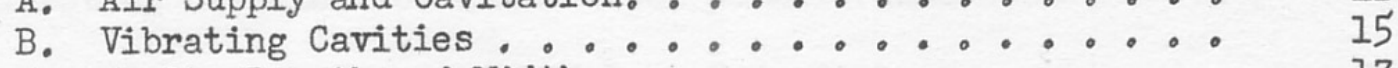

C. Cavity Length and Width. .................... 17

V. SUMMARY AND CONCLUSIONS. ................. 18

List of References ...................... 22

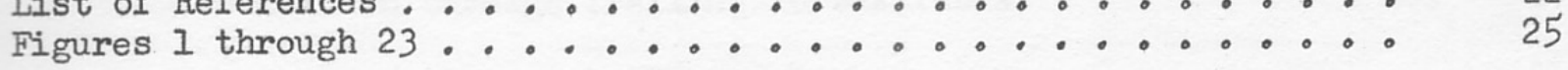




$$
\underline{I} \underline{I} \underline{S} \quad \underline{O} \underline{F} \quad \underline{I} \underline{L} \underline{L} \underline{U} \underline{S} \underline{T} \underline{R} \underline{A} \underline{T} \underline{I} \underline{O} \underline{N} \underline{S}
$$

Figure

1 The Two-Dimensional Free-Jet Water Tunnel. . . . . . . .

2 Schematic Drawing of Two-Dimensional Test Section and Appurtenances. . . . . . . . . . . . . . .

3 Typical Test Bodies. . . . . . . . . . . . . .

4 Comparison between Cavitation Number Based on Vapor

Pressure and Cavitation Number Based on Measured Cavity

Pressure .......................

5 Typical Pressure Records .................

6 Typical Nonvibrating Cavities..............

7 Air Supply Rate as a Function of Cavitation Number .....

8 Consecutive Motion Picture Frames for a Vibrating Cavity . .

9 Several Vibrating Cavities for a Two-Dimensional Normal

Plate. .......................

10 Several Vibrating Cavities for a Two-Dimensional Hydrofoil . .

11 Consecutive Motion Picture Frames for a Three-Stage Cavity . .

12 Vibrating Cavities for a Half-Span Normal Plate. . . . . .

13 Vibrating Cavities for a Half-Span Hydrofoil . .......

I4 Trailing Vortex Tubes....................

15 Ventilation through Trailing Vortex Tubes. ........

16 Ventilated Cavity in a Towing Tank ............ 38

17 Pressure Record during Change in Air Supply Rate . . . . . 39

18 Air Supply Rate for Two-Dimensional Bodies ......... 40

19 Air Supply Rate for Half-Span Bodies ........... 4 .

20 Effect of Jet Size on Air Supply Rate. . . . . . . . . 42

21 Cavitation Number for Each Vibrating Stage as a Function of

Initial Cavitation Number.................

22 Cavity Length as a Function of Cavitation Number . . . . . 44

23 Cavity Length for Vibrating Cavities . . . . . . . . 45 


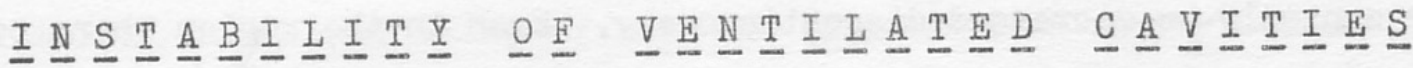

\section{INTRODUCTION}

It has been known for a long time that the characteristics of cavitating flows under steady conditions are mainly functions of the cavitation number. Cavitation number is here defined as

$$
\sigma=\frac{p_{0}-p_{k}}{\rho V^{2} / 2}
$$

where $\sigma$ is the cavitation number,

$\mathrm{p}_{0}$ is the undisturbed pressure at the body position,

$\mathrm{p}_{\mathrm{k}}$ is the cavity pressure,

$\rho$ is the liquid density, and

$V$ is the undisturbed velocity at the body position.

Equation (1) applies to supercavitating flows in which the cavity is longer than the body producingit and is more or less continuously filled with vapor or gas. Under natural conditions, the cavity pressure is approximately equal to the vapor pressure of the liquid and the cavitation number is determined mainly by $p_{0}$ and $V_{0}$. However, by introducing gas into the cavity it is possible to increase $\mathrm{p}_{\mathrm{k}}$ at will, decreasing the cavitation number and producing a "ventilated" cavity.

Natural and ventilated cavities should have similar average characteristics for a given body as long as the cavitation number is the same. It is sometimes advantageous to make use of this similarity and to produce cavities by ventilation. The purpose of the present investigation was to determine the gas flow requirements necessary to produce a ventilated cavity with a given cavitation number and to compare the cavity characteristics in natural and ventilated flows.

Surprisingly enough, it was soon found that the cavitation number could not be arbitrarily decreased by increasing the gas flow rate to a cavity. For a given natural cavity, there is a range of gas flow rates from zero upward where the cavitation number maybe more or less continuously decreased, but after a certain limiting gas flow rate has been reached, the cavitation 
number can only be decreased discontinuously. Even in the region where continuous decrease seems to occur, there may be minor discontinuities as the flow pattern changes with the addition of air. This paper is devoted to a presentation of the experimental relation between air supply and cavity behavior.

Other investigators [ 1 and 2] have already called attention to the anomaly in the relationship between rate of ventilation and cavitation number. Their work was done with circular disks placed normal to a horizontal water stream. The observation was made that once a certain air flow rate to the cavity behind the disks was exceeded, the cavity pressure increased very slowly thereafter. These investigators have attributed the slow pressure increase to rapid piping of the gas out of the cavity. The piping is supposed to occur through a pair of hollow trailing vortexes observable at the rear of the cavities, formed by circulation produced by gravitational effects on the horizontal cavity. However, it has been observed in the present experiments that the same rapid loss of gas occurs for nonlifting bodies in a vertical stream where there are no trailing vortexes. Even for lifting bodies where trailing vortexes do occur, it has been found that gas may be carried into, rather than out of, the cavities through the hollow vortexes. Hence, although the present research confirms the existence of the anomalies, it finds that they are more complicated than previously suspected and that the earlier explanation is not adequate.

This research has been supported by the Office of Naval Research of the U. S. Department of the Navy under Contract Nonr 710(24) Task NR 062-052. Messrs.C. H。 Su and F. Schiebe have assisted materially with the experimental observations.

\section{II。 EXPERTMENTAL APPARATUS}

The experimental studies were conducted in the two-dimensional, gravity-flow, free-jet water tunnel at the St. Anthony Falls Hydraulic Laboratory. The tunnel is a free-falling, nonrecirculating type utilizing river water and having a vertical test section. This tunnel has two principal features that make it especially useful for the type of investigation reported. herein. First, very low cavitation numbers can be obtained without blockage;

*Numbers in brackets refer to List of References on p. 22. 
and second, large quantities of air can be added to a cavity without having to remove the air from the tunnel water subsequently. Furthermore, the test sectionis completely transparent, facilitating visual observation and photography •

The tunnel test section is designed to produce a rectangular jet 5 in. thick and of variable width. The tunnel is shown in Fig, $I$ and the principal interior dimensions and some other features of the test section are illustrated in Fig. 2. For the present experiments the jet was operated at widths, $b$, of $6,8,10$, and 12 in, but mainly at 10 inches. The working velocity of the jet is between $20 \mathrm{fps}$ and $50 \mathrm{fps}$. Any pressure between vapor pressure and atmospheric pressure can be created at the test section. Velocity and pressure are controlled together by bleeding airinto the test-section space at both sides of the jet, while velocity alone can be controlled by a water inlet valve. Although the test section is designed mainly to accommodate two-dimensional test bodies of 5-in. span, it can also be used to study the effects of aspect ratio by inserting half-span bodies from one wall of the test section. The free-jet tunnel is described in detail in Reference [3].

Bodies of several different shapes were tested; these included modified Tulin-Burkhart low-drag hydrofoils [4], circular cylinders, and flat plates placed both normally and at small angles of attack to the stream. A.7l of the bodies are listed in the following table: they were 271 available in both full-span (two-dimensional) and half-span lengths. Typical test bodies in their mountings are shown in Fig。 3. All of the bodies were of stainless steel.

\section{TEST BODIES}

Designation

Normal Flat Plate

Circular Cylinder

Flat Plate Hydrofoil

Hydrofoil A

Hydrofoil B

Hydrofoil C

\section{Dimensions}

Widths: $B=1 / 8$ in., $3 / 16$ in。 Diameters: $D=1 / 8$ in., $1 / 4$ in., 0.54 in.

Chords: $c=1$ ino, 2 in.

Modified Tulin-Burkhart, 2-1/2-in。 chord, $\mathrm{C}_{\text {Id }}=0.1, a_{\mathrm{d}}=2$ degrees

Modified Tulin-Burkhart, 2-1/2-in。 chord, $\mathrm{C}_{\text {Id }}=0.2, \alpha_{\mathrm{d}}=2$ degrees

Modified Tulin-Burkhart, 2-1/2-in。 chord, $C_{I d}=0.3, a_{d}=3$ degrees
Number on Fig. 3

$$
\begin{array}{r}
2,5 \\
3 \\
6 \\
1,4
\end{array}
$$


Angle of attack, $\alpha$, of the hydrofoils is measured between the direction of the undisturbed stream and a straight edge laid against the pressure surface.

The methods of velocity $(V)$ and ambient pressure $\left(p_{0}\right)$ measurement are described in detail in Reference [3]. Essentially, velocity is determined by the pressure difference between the ring of taps seen near the top of Fig。 $I$ and the reference taps, while ambient pressure is measured by comparing the reference taps with the barometer. The cavity pressure $\left(p_{k}\right)$ was measured by means of a small steel tube inserted about $1 / 4$ in. below the body. Some of the tubes may be seen in Fig. 3. The plane of the open end of the tube is aligned with the flow. Fifty-inch mercury manometers were used for both average cavity pressure and average ambient pressure measurements and for measuring their difference. There was considerable fluctuation in the manometers when the pressures were low. The measured pressures inside natural cavities were generally slightly larger than the vapor pressure of the water. The discrepancy is greater, the greater the cavitation number: typical comparisons are shown in Fig. 4. The results agreewith those reported by Parkin and Kermeen [5] whose tests were conducted in a closed-jet water tunnel. The scatterin Fig。 4 at small cavitation numbers is probably attribo utable to the difficulties in reading the fluctuating manometers.

Pressure fluctuations inside the cavities were measured with electronic pressure transducers. Two were available, one being capable of measuring up to 25 and the other up to 10 psi fluctuation. The 25-psi transducer was mounted so that its face was flush with the inside rear wall of the test section on the centerline 6 in. below the test body center. Both transducers could also be connected to the test-section pressure taps shown in Fig. 2 and to two additional pressure taps, one at test body height and $I / 2$ in. to one side of the centerline and the other 9 in. below the test body center and 2 in. to the side of the centerline. Natural frequencies of the transducers were over $2000 \mathrm{cps}$ in air but were much lower in water and when coupled to pressure taps. Fluctuating pressures were amplified and were recorded by Brush recorders or by photographing the face of an oscilloscope. Typical. pressure records are shown in Fig. 5. Pressure records were obtained from the flush-mounted transducer whenever possible.

Air could be introducedinto a cavity through tubes which were built into the test bodies and also through the pressure taps in the rear test-section 
wall. The air tubes emerged from the trailing ends of the bodies and pointed downstream. Either room pressure or compressed air could be applied to the air intake. The rate of air flow was measured by a variable area flow meter. Examination of data shows that mean air velocities within the cavities were very small compared to the water velocities on the cavity wall.

Photographs were taken with a high-speed, $16 \mathrm{~mm}$, motion picture camera and with a 4 in. by 5 in. still camera from the front of the test section for full-span test bodies. Still camera pictures were exposed with a 10 or 20 microsecond flash lamp. For half-span test bodies, photographs were generally taken from the rear of the test section (the cavity side).

\section{BEHAVIOR OF VENTILATED CAVITIES}

A. Two-Dimensional Cavities

Natural cavities are characterized by a reentrant jet which moves through the interior of the cavity from the rear stagnation point toward the body, falls over to both sides, and returns to the stagnation point along the cavity wall which is a free streamline. When the cavity is short, the whole cavity appears milky, indicating that the reentrant jet reaches and hits the rear of the test body. When the caviky becomes sufficiently long, the reentrant jet does not reach the test body (because of energy dissipation along the free streamline where it mixes with the returning jet); this leaves the upper part of the cavity as a transparent space, filled largely with water vaporbut perhaps with other gases also. The cavity wall is clear above where the reentrant jet strikes and cloudy below. A natural cavity is illustrated by the photograph of Fig. 6a. The gases in the interior of the cavity are trapped as bubbles by the reentrant jet and carried down the sides of the cavo ity where many of them diffuse into the main flow and are carried into the wake. There, the vapor bubbles collapse and the gas bubbles pass on downstream。

Although the length of the reentrant jet fluctuates somewhat, natural cavities are quite stable. Neither pressure nor shape of the cavity alters appreciably with time if the flow is steady. The electronic pressure record obtained within a natural cavity is illustrated in Fig。 5c。 (Figures $5 \mathrm{a}$ and $5 \mathrm{~b}$ illustrate the pressure fluctuations in the wake of a body without cavity and in the wake of a cavity, respectivelyo) 
If a small amount of airfrom an external sourceis fed into a given cavity, the appearance of the cavity does not change very much. The size of the cavity is increased in accordance with the increase in internal pressure and the decrease in cavitation number, and the wake contains more air bubbles. The reentrant jet still appears, but it is somewhat shorter in length. Figure $6 \mathrm{~b}$ is a photograph of such a ventilated cavity, and Fig. 5d is its pressure record. Air may be added at ever-increasing rates without causing other types of changes until a certain critical cavitation number is reached. Until this critical is reached, the air supply rate is roughly proportional to the decrease in cavitation number as illustrated in Fig. 7 for a flat plate normal to the stream.

Once the critical cavitation number is reached, however, and air is added at still higher rates, several remarkable things happen. Visually, the cavity walls begin to vibrate violently, the cavity changing its length and width periodically, and the reentrant jet disappears for a part of the cycle. Figure 8 illustrates two cycles of the vibration as seen by a high-speed motion picture camera. An oscillographic pressure record was obtained at the same time as the pictures and is superimposed on them in Fig. 8--the pressure trace is recorded five frames earlier than the picture to which it pertains. Two still views of a similar vibrating cavity are shown in Fig.9a (where the pictures have been turned on their sides for convenience in mounting) and the corresponding Brush recorder record in Fig. 5e. Figure $5 f$ is the record for another such cavity. In the vibrating cavity air appears to be carried out of the cavity not as a continuous stream of bubbles, but rather as a mass of air-water mixture shed periodically from the end of the cavity.

Further increase in air supply rate, once the critical is reached, does not result in a corresponding increase in average pressure or decrease in cavitation number. This is shown by the data in Fig. 7. Rather, the cavitation number (and frequency of vibration) remains relatively constant while the cavity vibrates more violently. However, once a sufficiently high air flow rate is reached, the cavity suddenly increases in average length and pressure, and the cavitation number suddenly drops. The new cavity also vibrates, but less vigorously than the previous cavity, and its surface exhibits a wave form two waves in length; this may betermed a second-stage vibrating cavity (as opposed to the original first-stage cavity). Figure 9b illustrates a second-stage cavity and Fig. $5 \mathrm{~g}$ its pressure record. Further increases in 
air flow rate produce additional discontinuous changes in average cavity length, pressure, and cavitation number. As many as six stages have been identified for the two-dimensional, I/8-in. normal plate, and the first five of these are illustrated photographically in Fig. 9. Similar photographs for a hydrofoil are shown in Fig. 10. Figure 11 shows a high-speed film strip for a threestage cavity and Fig. 5i, a pressure record from the Brush recorder for such a cavity. The waves on all of these vibrating cavities are uniform across the thickness of the jet--they are two-dimensional.

As the number of stages and number of waves increase at a given ambient pressure and velocity, the wave lengths and amplitude of the pressure variation generally decrease. The transition between stages becomes less violent with increase in stage.

At high stages cavities frequently switch forth and back from one stage to another without measurable changes in cavitation number and waves are sometimes difficult to identify. At zero cavitation number (a splitcavity) there is no measurable pressure fluctuation (Fig. 5j) and there are, presumably, an infinite number of wave lengths.

The changes between stages just outlined do not necessarily follow in an orderly manner. Only if the air flow rate is increased very slowly is the orderly pattern the rule. Otherwise, the flow pattern can jump forth and back between stages andit can jump two or more stages at a time. The number of stages, and hence, the cavitation number, at a given air flow rate depends on many factors, but the main ones seem to be the size of the initial cavity before air was added and the rate of change of the air supply. The data in Fig. 7 illustrate some of the possibilities. Data for three different natural initial cavities are shown, that for the largest being at the lower left and for the smallest at the upper right. As a matter of fact, the smallest cavity only exists when air is supplied and the concept of an initial cavity with zero air is purely hypothetical in this case.

It is not possible to go through a large number of stages for a given initial cavity in the free-jet tunnel because of the limited test-section length. Once the end of the cavity approaches the end of the test section (about 36 in. from the body) the cavity is ventilated to the atmosphere surrounding the jet--it splits--and the cavitation number is reduced to zero. 
To achieve a cavity with a large number of stages in this tunnel it is necessary to start with a very large hypothetical initial cavitation number; this causes the first real cavity to occur in the second or higher stage, as explained later in Part IV-C.

\section{B. Cavity of Finite Span}

A question arises as to whether the behavior just described may be characteristic of two-dimensional cavities only. Tests of the half-span bodies answer this question in the negative.

For nonlifting bodies such as the normal flat plate and circular cylinder there is little difference between the two-dimensional and finite aspect-ratio case. What difference there is appears as a somewhat smaller air requirement per unit span to produce a given ventilated cavity from a given initial cavity and a slightly shorter cavity length. The reduced air requirement and length seem to be associated with the fact that the span of the cavity near its downstream end is less than that of the test body in the finite aspect-ratio case, while in the two dimensional case the cavity span is constant. Figure 12 shows some vibrating cavities for the half-span flat plate placed normally to the stream, together with the pressure records. Similar data for a lifting body are shown in Fig. 13.

In the case of lifting bodies, a trailing vortex appears at the free end of the span. The trailing vortex extends far downstream and, at low pressures, it is marked by at least a very fine stream of air bubbles in its center. Below the test section the trailing vortex is frequently ventilated by the turbulent air-water mixture flow in the tunnel discharge pipe, and becomes hollow for part or all of its length. Ventilation is usually intermittent. Several stages of vortex ventilation are shown in Fig. 14. The only air reaching the cavities in Fig. I4 comes upthrough the hollow vortexes; ventilation ceases after the hollow vortexes are closed. When a hollow vortex reaches a natural cavity, the cavity suddenly enlarges, and usually with a bang.

Figure 15 illustrates the interaction of vortex tubes with ventilated. cavities. On the left side of Fig. 15a, where the vortex has not quite reached the body, there is no ventilation other than that occurring through the air inlet tube in the trailing edge of the hydrofoil. In the two center pictures air coming through the vortexes has enlarged the free ends, only, of the ventilated cavities, while on the right side of Fig. $15 \mathrm{~b}$ air from the vortex is 
distributed throughout the cavity. At no time in the present experiments was air observed to travel out of the cavity and down the vortex. In fact, it is believed that the vortexes contributed to the air supplied to the cavities (and this air could not be metered).

Except for the presence of trailing vortexes and shortened cavity spans near their downstream ends, cavities from finite-span lifting bodies look exactly like the two-dimensional cavities in both the steady and vibrating regimes.

\section{Boundary Effects}

Since the present experiments were conducted in a vertical free jet, a question may arise as to whether the peculiar vibrating conditions are associated with the tunnel geometry. This question is best answered by comparison with towing tank results in which the cavity is horizontal and there is only one free surface. Towing tank experiments are also underway at the St. Anthony Falls Hydraulic Laboratory under another research contract. These show similar wave forms and the same fluctuating pressures associated with cavities as were found in the free-jet tunnel. Figure 16, taken from a preliminary report on the towing tank work [6], illustrates this point。

\section{Details of the Vibrating Motion}

The mechanism by which cavity vibration is established is not known with certainty as yet, but it must be associated with the presence of air in the cavity. It has been impossible to establish vibration in a cavity with too little air, regardless of how long the cavity has been made (up to the 36-in. length available). But with sufficient air, even very short cavities ( 4 in. or less) can be made to vibrate. The method of air inlet (number, shapes, and size of inlets) makes no difference in behavior, indicating that the cavity is not acting like an elastic Helmholtz resonator. Vibration frequency does seem to depend on cavity length, however, the frequency increasing for shorter cavities.

Much has been learned about the details of the vibrating motion by examining combined motion picture and oscilloscopic records like those shown in Figs. 8 and 11. Referring to Fig. 8, in particular, the process appears to be as follows: 
In frames 1 to 11 , where the cavity is stretching, the pressure is falling. This indicates that the air supply rate is not sufficient to maintain the increasing cavity volume. Because of the falling pressure, cavitation number is rising. For steady cavities, larger cavitation numbers are associated with shorter and thinner cavities. Apparently, in the unsteady case represented by Fig. 8 the rising cavitation number requires a smaller cavity also, and by the time frame 12 (starred) is reached, the required cavity length just equals the length to the narrow neck of the cavity. This neck then closes and a reentrant jet forms, shooting waterback into the cavity. Cavity pressure now increases because the inflow of water from the trailing edge and air from the body are together more than sufficient to fill the cavity. The pressure increase is transmitted with the speed of sound in the cavity atmosphere to the head end of the cavity. With increasing pressure, the cavitation number decreases and the cavity must become both longer and wider; the widening process is visible in the figures following frame 12. At the same time, air bubbles are being removed from the cavity by the same process as in the steady flow case, and the accumulation of turbulent jet fluid at the trailing end of the cavity is causing an energy loss in the free streamline. Because of the energy loss, the length of the reentrant jet gradually decreases and by the time frame 22 or 23 has been reached (black circle) the reentrant jet has practically ceased. But the cavity is lengthening to accommodate the new cavitation number which was determined at the test body. With little new water being added, the lengthening process again reduces the pressure. The falling pressure is transmitted to the head of the cavity where the rising cavitation number now decrees a narrower and shorter cavity and the process repeats itself. At the time that the trailing end of the cavity is sealed, the previous wave consisting of a mixture of water (from 
the reentrant jet) and air moves off in the wake at tunnel speed leaving a conspicuous gap at the new trailing edge of the cavity. (There are some bubbles in the gap, but these have come down the sides of the cavity from the reentrant jet entraining process.) It is this pinchingoff process which enables the vibrating cavity to carry off so much more air than the steady cavity.

The process just described can also be followed in the three-stage cavity of Fig. 11 where the stars and circles have the same meaning as in Fig. 8. Each wide and narrow portion of the cavity was generated by a high or low pressure in the cavity at the test body. The speeds of the wide and narrow portions of the cavities have been measured from the motion picture records. For third-and higher-stage cavities, the waves move uniformly at cavity speed $(V \sqrt{1+\sigma})$, but for first- and second-stage cavities the speed is less, being as little as half the cavity speed for some first-stage cavities. The wide and narrow portions of a cavity, once generated, maintain their widths for one or more waves, depending on the number of stages.

At high air flow rates and with a cavity of multiple stage, sealing does not always occur at the same place. Pressure records, as in Fig. 13c, sometimes show consistent sealing for a number of cycles and then an interval in which vibration does not occur at all and the cavity becomes temporarily very long. Usually sealing will resume again at the oxiginal place, but if it does not, the cavity splits permanently and the cavitation number goes to zero. A permanent change of stage occurs by a similar process, the point of sealing occurring after the $n \pm 1$ wave instead of after the nth wave. The mechanism determining the number of stages is not known.

Pressure records obtained from two transducers attached to identical pressure taps 6 in. apart along a cavity show no phase differences and the same amplitudes for a variety of vibrating cavities. Vibrating pressures within the cavity are actually transmitted in phase, but at reduced amplitude, throughout the stream and even to the surrounding atmosphere (which is limited by the test-section walls). Figure $5 f$ shows the pressure vibration in the liquid close to the side of a cavity. (Because measurement was made through a pressure tap filled with liquid, a direct amplitude comparison with Fig. 5g, which was made by a flush-mountedtransducer acting against air and vapor, is not possible.) For this cavity, produced in a $10 \mathrm{in}$. wide jet, the pressure 
amplitude in the atmosphere outside the jet is about $1 / 10$ or less of that within the cavity. (For an equally long nonvibrating cavity, there is no detectable vibration of commensurate frequency in the atmosphere outside the jet.) The in-phase pressure vibration in the jet reduces but does not eliminate the net change in cavitation number at the body from that implied in the previous discussion.

Figure 17 shows the pressure record obtained while a vibrating cavity was first reduced in length by suddenly closing off the air and then increased in length by suddenly reopening the air. When the air supply is shut off, the cavity eventually stops vibrating as shown by the record. As the cavity shortens, its trailing edge passes over the pressure transducer producing a pressure near stagnation (considering the energy loss along the free streamline), and finally the pressure transducer is left in the cavity wake where the pressure is tunnel ambient pressure. On reversing the process, a very similar record is traced in the opposite direction.

\section{EXPERIMENTAL RESULTS}

A. Air Supply and Cavitation Number

Examination of many graphs like the one in Fig. 7 shows that for a given body the air required to produce a given cavitation number is solely dependent on the initial cavitation number. As already mentioned in connection with the discussion of Fig. 7, the initial natural cavity is frequently hypothetical only, no cavity existing untilair is introduced. In such cases,

$\mathrm{p}_{\mathrm{k}}$ in Eq. (I) cannot be measured for the initial cavity. Even when $\mathrm{p}_{\mathrm{k}}$ can be measured for a natural cavity, it has a variable relation to the vapor pressure as indicated by Fig. 4. Henceforth, to define an initial cavitation number that is universally useful it is assumed that $p_{k}=p_{v}$, the vapor pressure of the liquid, and the initial cavitation number is defined as

$$
\sigma_{\mathrm{v}}=\frac{p_{0}-p_{v}}{\rho V^{2} / 2}
$$

All of the air supply data obtainedin the present experiments have been shown in Figs. 18 and 19 by plotting the weight rate of air supply per 
unit span, per unit chord or width, $\mathrm{w}_{\mathrm{A}}$, against the parameter $\sigma / \sigma_{\mathrm{v}}$. Figure 18 is for two-dimensional bodies and Fig. 19 for half-span bodies. It is seen that in the subcritical region, the data on each graph tend to follow one of two or three straight lines, data for the cavities with largest $\sigma_{v}$ falling on the line of greatest slope. In the supercritical region, the data fall on definite values of $\sigma / \sigma_{v}$, each value corresponding to a specific cavity stage. It is believed that there would be a quite sharp break between the subcritical and supercritical regions except for the fact that cavities near critical vibrate some of the time and are steady some of the time.

It is readily apparent from these graphs that the entire effect of scale for similar bodies is accounted for by measuring the air flow rate on a unit span and a unit chord or width basis.

Observation of the flow has produced the following explanation for the three groups of data in the subcritical regime. The lowest set of data points on each graph is associated with cavities in which the reentrant jet does not reach the air inlet tubes. Thus, air is entrained onlyby the splashing of the reentrant jet. The next higher set of data is produced when the reentrant jet does reach the air inlets (usually when the jet strikes the body). As a result, air is entrained more rapidly by the jet and diffused more rapidly to the surrounding stream。 When an attempt is made to increase the length of a cavity from just under to just over the reentrant jet length, there is a small discontinuity in cavity length and $\sigma$, and the data points in the graphs of Figs. 18 and 19 jump from the middle line to the lower line. (A similar trend in data was shown by Campbell and Hilborne [2] in the vero tilated circular disk.) There may also be a small hysteresis loop in the reo gion of the discontinuity.

The uppermost line of datain Figs. 18 and 19 results when there is no true continuous cavity, and what would otherwise be the cavity region is occupied by a mixture of numerous small cavitation and air bubbles in water. Air is carried away directlyby the stream and entrainment is greater than in either of the other two processes. Although the data that form the upper lines on the graphs are not associated with true cavities, they are included on some of the graphs because they complete the picture. Again, there is a small discontinuity and hysteresis loop when the cavitation number change is such as to cause the data to jump from the upper to the middle line, or vice versa。 
In Fig. 18, straight lines have been drawn by eye through the two lower groups of subcritical data of each graph. On the cambered hydrofoil graphs ithas been possible to approximate these lines by the empirical formulas

$$
\begin{aligned}
& \mathrm{w}_{A}=4.8 \times 10^{-4}\left(0.9-\sigma / \sigma_{\mathrm{V}}\right) I \mathrm{c} \sin \alpha_{,} \quad 0<\sigma_{\mathrm{V}} \lesssim 0.2 \\
& \mathrm{w}_{\mathrm{A}}=8.6 \times 10^{-4}\left(0.9-\sigma / \sigma_{\mathrm{V}}\right) \mathrm{L} \mathrm{c} \sin \alpha_{,} \quad 0.2 \lesssim \sigma_{\mathrm{V}} \lesssim 0.5
\end{aligned}
$$

where $w_{A}$ is the air flow rate in lbs per sec $\left(w_{A}=I c W_{A}\right)$,

II is the span length in inches,

$c$ is the chord length in inches, and

$a$ is the angle of attack measured from the chord line.

Equations (3) and (4) have also been drawn for reference on the flat plate hydrofoil graph. These equations serve to show that the air required for ventilating a hydrofoil to produce a given cavitation number under given flow conditions is proportional to the angle of attack, chord length, and span length of the hydrofoil and is nearly independent of camber. The limits on $\sigma_{v}$ givenwith the formulas are very rough and may change with scale or boundary conditions. Formulas comparable to Eqs. (3) and (4) for the normal plate and circular cylinder have been written on the corresponding graphs of Fig. 18.

The straight lines obtained from the two-dimensional subcritical data have been superimposed on the corresponding halfospan graphs of Fig. 19 for comparison. Examination shows that finite-span, nonlifting bodies require less air than their twomdimensional counterparts, perhaps because of the reduced cavity span at the trailing end. Iifting bodieswhich have trailing vortexes appear to require even less air compared to their two-dimensional counterparts; it is believed that the difference is attributable to inflow of unmetered air through the hollow vortexes.

In the graph for hydrofoil A at 12 -degree angle of attack in Fig. 19, data from the towing tank for the same hydrofoil [6] have been plotted along with the tunnel data. The hydrofoils had the same aspect ratios in both experiments, but, whereas the tunnel foil had a half span of $2-1 / 2$ inches in the 5-in. thick jet, the towing tank foil had a full span of 5 inches in a 
9-ft wide tank. The tunnel foil was submerged 5 in. on both sides while the towing tank foil was submerged $6 \mathrm{in}$. on the cavity side only. It is apparent that the tank data were all obtained for vibrating cavities of high stage and that they are quite comparable to the tunnel data.

The tunnel data presented in Figs, 18 and 19 were obtained in a 10-in. wide jet. Figure 20 contains typical supplementary data taken with a view toward examining the effect of jet width on the experimental data. The graphs presented are representative of all the data and indicate that there is no measurable effect of jet width.

\section{B. Vibrating Cavities}

If the cavitation number corresponding to a first-stage vibrating cavity is designated $\sigma_{\mathrm{cl}}{ }^{2}$ to the second stage, $\sigma_{\mathrm{c} 2}$, and so on, it is remarkable that $\sigma_{c} / \sigma_{\mathrm{V}}$ for each stage in Figs。 18 and 19 appears to be nearly constant for all bodies. A special effort has been made to measure $\sigma_{c}$ as a function of $\sigma_{\nabla^{\circ}}$ Typical data (which are in addition to those shown in Figs. 18 and 19) are shown in Fig. 21. The data in Fig。 21 were obtained by producing a cavity of given stage and then increasing $\sigma_{\nabla}$ gradually until it was impossible to maintain that stage any longer。

The data of Fig. 21 confirm the fact that there is a unique relao tion between $\sigma_{c}$ and $\sigma_{\nabla}$ for each stage, and that this relation is nearly the same for all bodies independently of shape, size, angle of attack, and aspect ratio. For small cavitation numbers, the ratio of $\sigma_{\mathrm{C}} / \sigma_{\mathrm{V}}$ is virtually constant as indicated by the straight lines drawn on the graphs, but at higher cavitation numbers the ratio decreases from its constant value. It should be observed that if firstestage cavities are omitted from considera. tion, the slopes of the straightlines in Fig. 2la satisfy the empirical formo u.la

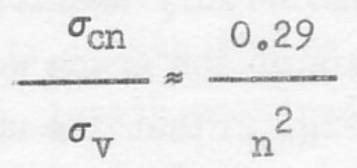

where $n$ is the number of stages. The same formula applies well enough to the other graphs in Fig. 21, also. A sampling of towing tank data [6] indicates that Eq。 (5) is also applicable in the towing tank. The data given in 
connection with Fig. 16, for example, lead to an expectation of six stages; a count of the number of waves leads to this same number. Likewise, Eq. (5) was applicable to test data from jets of other widths than 10 inches. It may be supposed that a formula of the form of Eq. (5) is universally applicable for other than first-stage vibrating cavities at small cavitation numbers. For first-stage cavities

$$
\sigma_{\mathrm{cl}} / \sigma_{\mathrm{v}} \approx 0.19
$$

is the corresponding formula where a constant ratio is applicable。

Equation (5) gives no information as to which stage or which $\sigma_{c}$ will occur for a given $\sigma_{v^{\circ}}$ As already observed in the discussion in Part III, vibration may occur at any one of several stages, depending on how carefully the air is added. In Fig. 2la for the two-dimensional normal plate, for example, up to $\sigma_{v}=1.25$ any stage of vibration from the first up could occur. (The lowest data point on eachline simply indicates the longest cave ity that could be obtained in the free-jet tunnel without splitting the cavity. If the tunnel test section were infinitely long, the data could have been extended to the origin along all lines。) On the other hand, a firststage cavity could not be maintained above $\sigma_{\nabla}=1.25$ even though the cavity wasstill of the order of 7 in. long; only nonvibrating cavities or secondw or higher-stage vibrating cavities could be produced then, no matter how carefully the air was added。Similarly, above $\sigma_{v}=3.1$ vibrating cavities were possible only for third or higher stages. The shading in Fig。2la shows the limitations on several stages (although the limitations on the highest-stage cavities are again uncertain because of the physical limits of the tunnel)。 Similar shading could have been added to all of the graphs in Fig。2l. It can be seen that the shading on all of the graphs for two-dimensional bodies would be nearly congruent, but for the half-span bodies, the upper limitation on $\sigma_{\nabla}$ at each stage was considerably smaller than for the two-dimensional bodies, and the vibrating portion of the graph would be correspondingly smaller. Althoughit appears from Fig. 2l that the upper limit on $\sigma_{v}$ is strongIy dependent on aspect ratio, the towing tank data [6] for the same aspect ratios gave higher $\sigma_{v}$ for a given stage than did the half-span bodies. It appears, therefore, that the test facility mayhave some influence on the maximum $\sigma_{v^{\circ}}$ Nevertheless, it is believed that the concept of a maximum possible $\sigma_{\mathrm{v}}$ for each stage, as suggested by the shading in $\mathrm{Fig}$. 2la, is valid. 
In fact, in the towing tank tests as illustrated by Fig. 16, cavities always occurred with a large number of wave lengths and it is thought that this was true because of the high initial cavitation numbers.

\section{Cavity Length and Width}

Some typical cavity length measurements are presented in Fig。 22 for both two-dimensional and half-span bodies. The lengths, $\ell$, are made dimensionless with reference to the appropriate linear dimension of the test body. These data were obtained for both natural and ventilated, nonvibrating cavities, as indicated on the graphs. For vibrating cavities the length is variable, as already noted, and the data are considered subsequently.

Several facts are apparent from Fig. 22。 First, cavity length appears to be a unique function of cavitation number regardless of whether the cavity is natural or ventilated. Second, as already mentioned in Part III, half-span bodies have shorter cavities than two-dimensional bodies。 And third, relative jet size has no important effect except in the case of the circular cylinder; on the cylinder, the narrower the jet, the shorter the cavity。 The jet-size effect on the cylinder may be attributable to a shift in separation point on the cylinder--all of the other test bodies are sharpaedged so that the separation point cannot shift.

Cavitywidth measurements (not presented here) show that width, also,

is a unique function of cavitation number. Cavity width at the plane of symmetry of a half-span body is little different from that of its twomdimensional counterpart.

For a vibrating cavity, cavitation number as well as length actuo ally varies during a cycle. Cavity pressure as measured by the average read. ing of the mercury manometer has been tacitly accepted as the desired pressure in measuring cavitation number. It would be desirable to have an equally simple measurement for cavity length. On the high-speed motion pictures, both a minimum and a maximum cavity length can be measured. The minimum length appears to be closest to the length measured for nonvibrating cavities and some results of the measurement of minimum length for the normal plate are shown by circles on Fig. 23. Multiplying the wave length by the number of waves or stages yields a length comparable to the minimum length measured on the motion pictures; the latter length is available from still pictures as 
well as motion pictures and some typical results are shown by square symbols on Fig. 23. There is a good deal of spread in the length measurements, but it appears that the shortest length of a vibrating cavity is comparable to the length of a nonvibrating cavity at the same average cavitation number: (It may be recalled that a reentrant jet is associated with a vibrating cavity when it is short but not when it is long. A reentrant jet is also an essential part of a steady cavity.) The triangles on Fig. 23 represent estimates of the average shortest cavity length obtained visually using stroboscopic illumination in the tunnel, and these measurements, too, seem to be in good agreement with those for nonvibrating cavities. A result similar to that shown in Fig. 23 was obtained for the cavities produced by bodies other than the normal plate.

The widths of vibrating cavities are so variable, even from one wave length to the next, that no attempt has been made to analyze the pictures for cavity width。

\section{SUMMARY AND CONCLUSIONS}

When air is supplied to the wake of a moving body, an artificial or ventilated cavitymay be created. There are two distinctclasses of ventilated cavities, however. One class may be called steady and the other vibrating.

A steady cavity occurs at the smaller airflow rates and is similar to a natural cavity in all respects. Cavitation number can be very effectively reduced by adding air to a wake in this regime. The air required is nearly directly proportional to the reduction in cavitation number. Pertinent air supply datafor vertical cavities are given in Figs, 18 and 19, and these data may be reasonably extrapolated to larger and smaller bodies than those tested in the present program. For two-dimensional hydrofoils, the approximate empirical formulas

$$
\begin{aligned}
& w_{A}=4.8 \times 10^{-4}\left(0.9-\sigma / \sigma_{V}\right) L c \sin \alpha, 0<\sigma_{V} \lesssim 0.2 \\
& w_{A}=8.6 \times 10^{-4}\left(0.9-\sigma / \sigma_{V}\right) I c \sin \alpha, 0.2 \lesssim \sigma_{v} \lesssim 0.5
\end{aligned}
$$

may be used for this extrapolation where 
$\sigma$ is the cavitation number defined by Eq. (1) on p. I,

$\sigma_{V}$ is the initial cavitation number defined by Eq. (2) on p. 12,

$\mathrm{w}_{\mathrm{A}}$ is the air flow rate in lbs per sec,

$I$ is the span length in inches,

c is the chord length in inches, and

$\alpha$ is the angle of attack measured from the chord line.

For finite-span foils, the air flow rate is somewhat smaller. It is believed that horizontal cavities would behave similarly to the vertical cavities which were tested.

The Eqs. (3) and (4) represent two subclasses of steady cavities. The first pertains to cavities whose reentrant jet does not reach the air inlets to the cavity while the second pertains to cavities in which the jet does reach the air inlets. The limits given for these equations probably vary somewhat with the scale and orientation of the body and with the external flow boundaries. If $\sigma_{\mathrm{V}}$ exceeds the upper limit of Eq. (4), no true, steady cavity is possible; instead, the body wake is filledwith a mixture of small bubbles and water.

A vibrating cavity is formed when too much air is added to the cavity--that is, when the cavitation number is reduced below a certain critical value. It has been found by experiment that the maximum value of this critical is 0.19 of the initial cavitation number $\left(\sigma \leq 0.19 \sigma_{v}\right)$. For cavitation numbers higher than this critical no vibration can occur, under any circumstances.

A vibrating cavity has a wavy surface (with wave fronts normal to the flow direction), a periodically variable length, and a periodically fluctuating internal pressure. Depending on whether there are one, two, or more waves in the surface, such a cavity may be termed first-stage, second-stage, and so on. If air is added slowly to a cavity of sufficiently small initial cavitation number, the first vibration after passing through critical will be a first-stage vibration. Once this vibration occurs, additional air will not necessarily produce a further reduction in cavitation number. The only wayto produce a lower cavitation number is to create a second- or higher-stage cavity. This can be done by increasing or decreasing the air flow rate rapidlyor by starting with a higher initial cavitation number. When a higher-stage 
cavity is produced from the next lower stage, the cavitation number jumps discontinuously to the lower value corresponding to the higher stage. There appears to be a fixed ratio between the cavitation number corresponding to each stage and the initial cavitation number, the ratio being determined mainly by the stage. Both two-dimensional and finite aspect-ratio bodies, lifting and nonlifting bodies, regardless of shape, fit the same empirical formula for this ratio; the formula is given as Eq. (5) on p. 15.

If a ventilated cavity is produced by starting with a high initial cavitation number, vibration cannot begin with the first stage, but will beginwith some higher stage. Figure 2la illustrates the limitations on vibration. The transition between vibrating stages is quite erratic. Sometimes the stage, and with it the cavitation number, changes with little or no apparent reason. This is especially true at the higher stages where the vibration is not so pronounced as at the lower stages. For this reason, it is not possible to establish a definite relation between air supply and cavitation number in the vibrating regime. The air requirements are generally so large compared to those in the steady regime that it could hardly be considered practicable to reduce cavitation number by adding air once vibration sets in. However, experimental data that can be extrapolated to other body sizes and shapes are given in Figs. 18 and 19.

If the length of a vibrating cavity is defined as its shortest length during a cycle, the lengths of all cavities--natural and ventilated, steady and vibrating--are the same function of average cavitation number. This function is shown for various bodies in Fig. 22. Vibrating cavities at their longest are, of course, one wavelength longer than given in Fig。22. Finite span cavities are slightly shorter than two-dimensional cavities at the same cavitation number。

The exact cause for cavity vibration is still poorly understood. A detailed description of the vibration phenomenon was presented in Section III-D. There is a fundamental difference in the method of air removal from vibrating and steady cavities. In the latter, air is removed entirely by en. trainment in the reentrant jet and diffusion from the jet through the adjacent cavity walls. In the vibrating cavity, the reentrant jet is intermittent and carries off only a small part of the air. The bulk of the air leaves as an air-water mixture once each period as successive waves are pinched off at the 
end of the cavity.

A division between ventilated cavity regimes similar to that given hereinwas discussed in References [1] and [2]. It is possible that the "trailo ing vortex regime" described in those references is the vibrating regime of the present paper, and that the mechanism of air outflow is really the same in the two cases, even though the authors of those papers attributed the change in regime to rapid outflow of air through the hollow trailing vortexes. (Actually, it was found in the present experiments that air tends to flow into a cavity through the trailing vortexes.) As already stated, for high-vibration stages it is neither easy to recognize the vibrations by eye nor to separate one stage from another, so that the vibration phenomenon could easily have been missed in the experiments of References [1] and [2]. As a matter of fact, one of the discussors of Reference [1] called attention to what appeared to be vibrating cavities in motion pictures shown at the time of presentation: the author in his reply stated that the vibration was probably attributable to the tunnel circuit.

Some preliminary towing tank data for cavities produced by ventilating hydrofoils were presented in Reference [6], and these agreed with the present tunnel data in all respects where the data overlap--in the vibrating regime. The strange twistings of the air supply curves in Reference [6] can be understoodin terms of the changes in stage of the cavities. It is apparent that because of the high initial cavitation number, all of the data in Reference [6] are perhaps at the fifth or higher stage of vibrating cavity. 


\section{$\underline{I} \underline{I} \underline{T} \quad \underline{O} \underline{F} \quad \underline{R} \underline{E} \underline{F} \underline{E} \underline{R} \underline{E} \underline{N} \underline{C} \underline{E} \underline{S}$}

[1] Cox, R. No, and Clayden, W. A. "Air Entrainment at the Rear of a Steady Cavity." Proceedings of the N. P. L. Symposium on Cavitation in Hydrodynamics. London, 1955. Paper No. 12, 19 pages.

[2] Campbell, I. J., and Hilborne, D. V. "Air Entrainment Behind Artificially Inflated Cavities." Second Symposium on Naval Hydrodynamics. Washington, D. C., August 1958. Proceedings in print.

[3] Silberman, E., and Ripken, J. F. The St. Anthony Falls Hydraulic Laboratory Gravity-Flow Free-Jet Water Tunnel. University of Minnesota, St. Anthony Falls Hydraulic Laboratory Technical Paper No. 24, Series B. April 1959. 45 pages。

[4] Tachmindji, A. J., Morgan, W. B., Miller, M. L., and Hecker, R。 The Design and Performance of Supercavitating Propellers. DavidTayIor Model Basin, Report C-807. February 1957. 31 pages.

[5] Parkin, B. R。, and Kermeen, R。 W。 "Water Tunnel Techniques for Force Measurements on Cavitating Hydrofoils." Journal of Ship Research, Vol. I, No. I. 195 pages.

[6] Wetzel, J。M. Artificial Cavities on Submerged Hydrofoils of Finite Span. University of Minnesota, St。 Anthony Falls Hydraulic Laboratory Memorandum No. M-78. May 1959. 36 pages. 
$\underline{F} \underline{\underline{G}} \underline{\mathrm{U}} \underline{\mathrm{R}} \underline{\mathrm{E}} \underline{\mathrm{S}}$

(I through 23) 


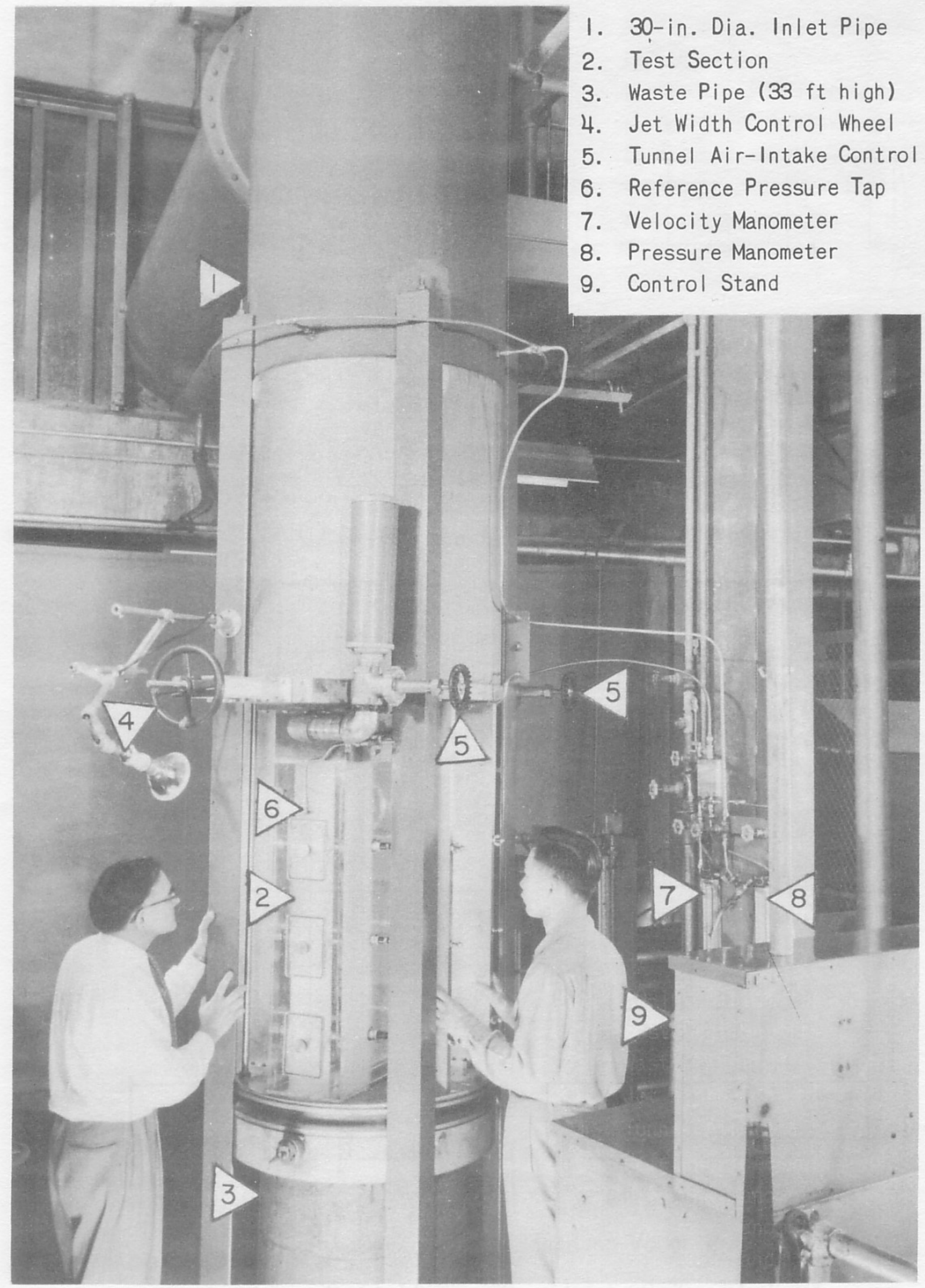

Fig. 1 - The Two-Dimensional Free-Jet Water Tunnel 


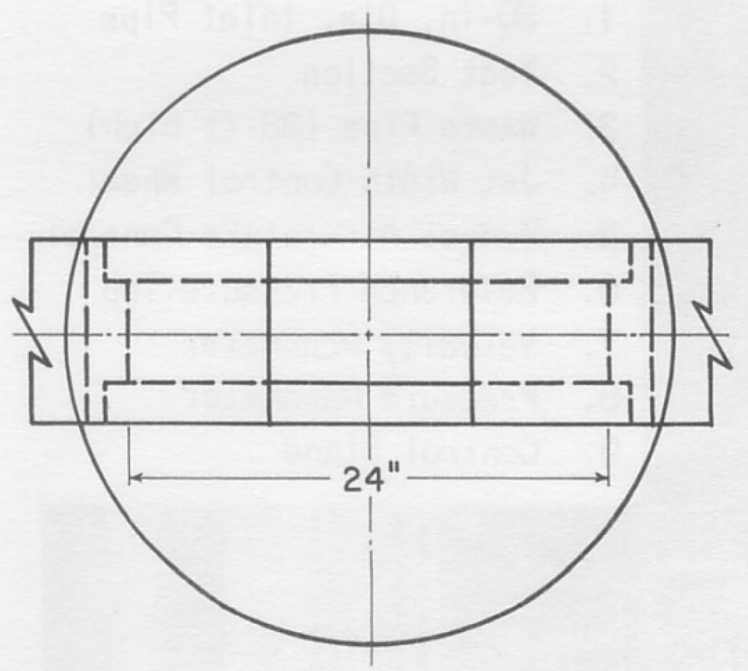

\begin{tabular}{ccc}
\multicolumn{3}{c}{$\begin{array}{c}\text { Width of Jet } \\
\text { in Inches }\end{array}$} \\
B & $b_{\infty}$ & \multicolumn{1}{c}{$b$} \\
8.06 & 5.00 & 5.00 \\
9.63 & 6.00 & 6.01 \\
12.63 & 8.00 & 8.03 \\
15.47 & 10.00 & 10.06 \\
18.09 & 12.00 & 12.15 \\
21.62 & 15.00 & 15.28 \\
22.73 & 16.00 & 16.36
\end{tabular}
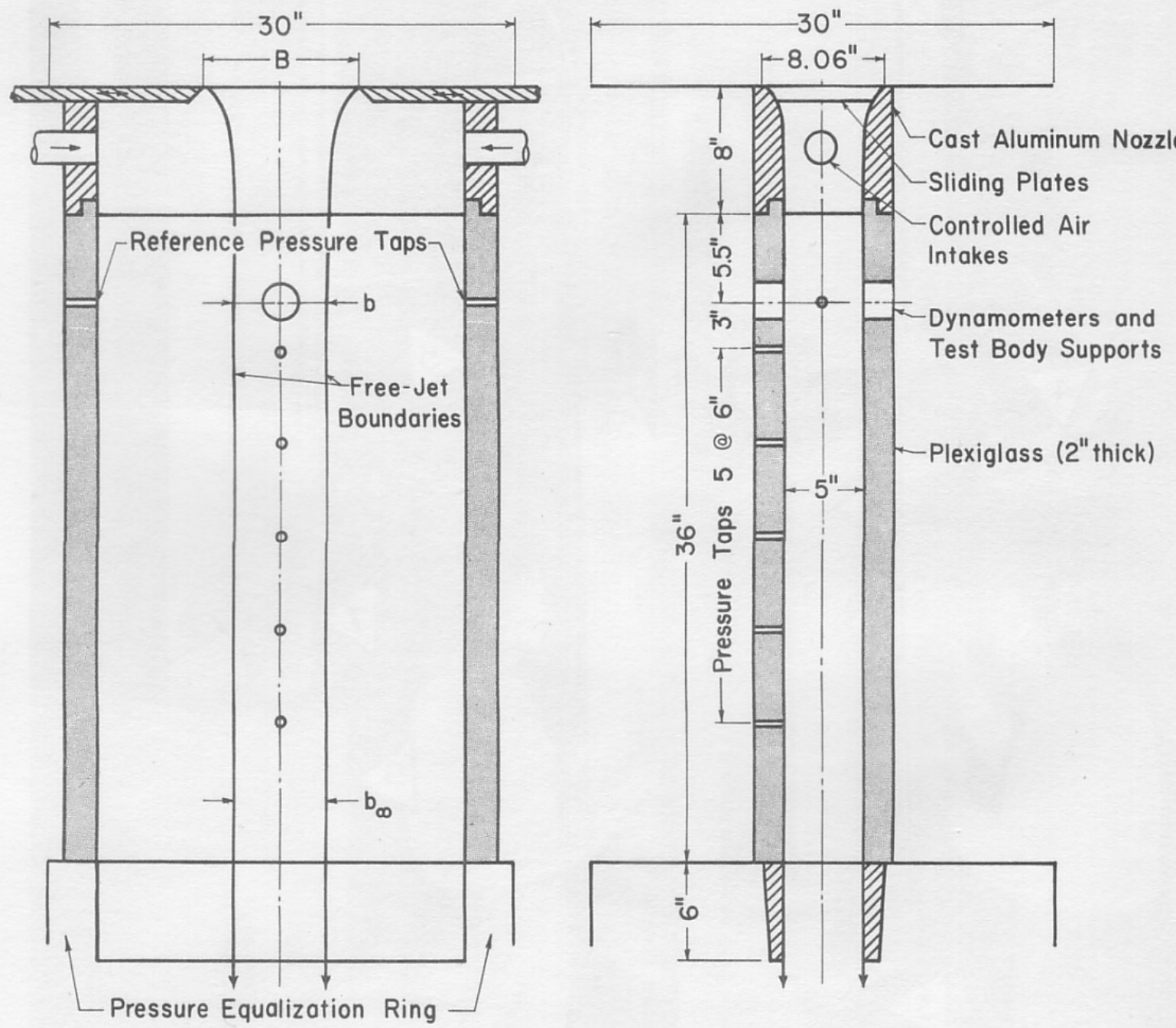

Fig. 2 - Schematic Drawing of Two-Dimensional Test Section and Appurtenances 


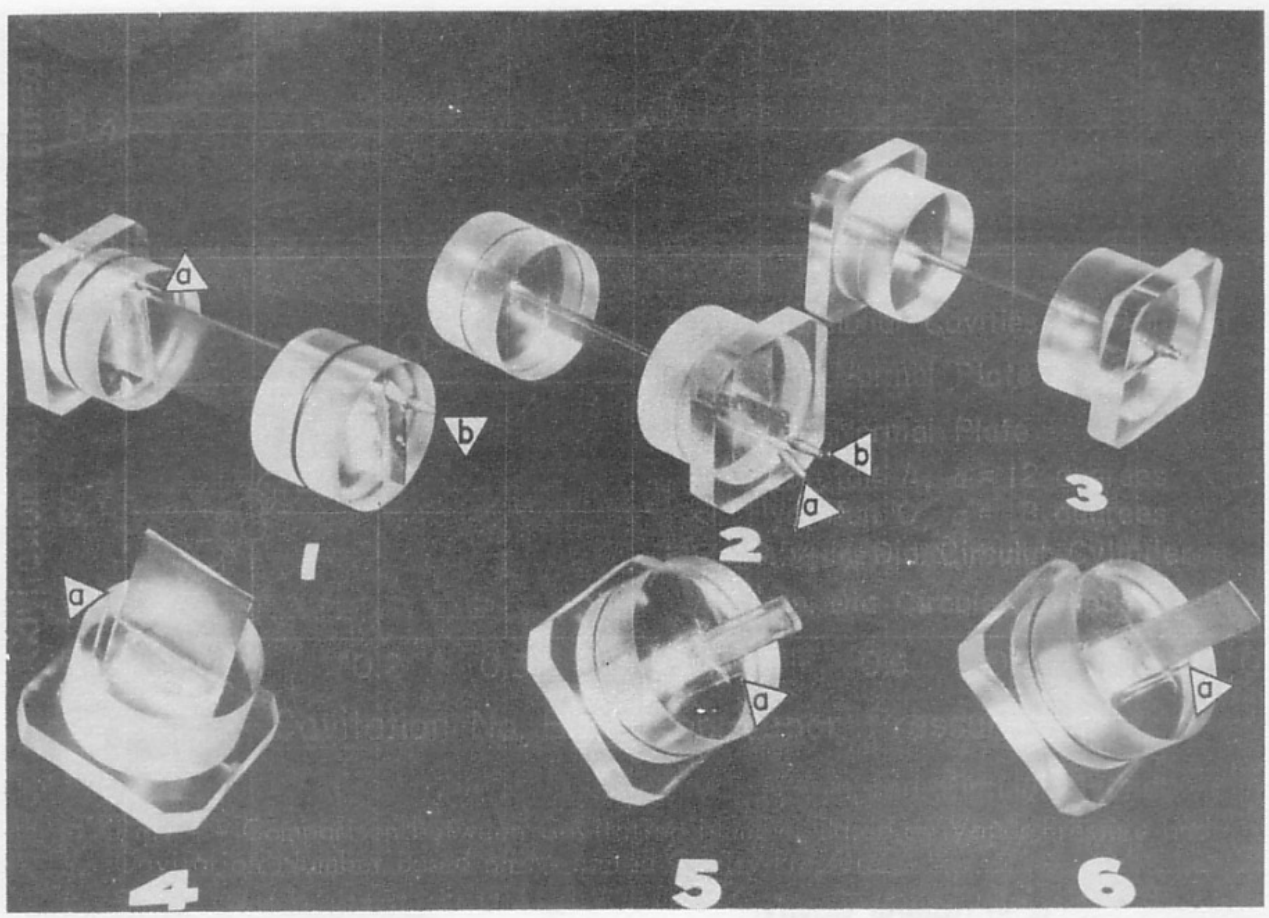

$a-$ Cavity Pressure Measuring Tube

b - Air Supply Tube

Fig. 3 - Typical Test Bodies

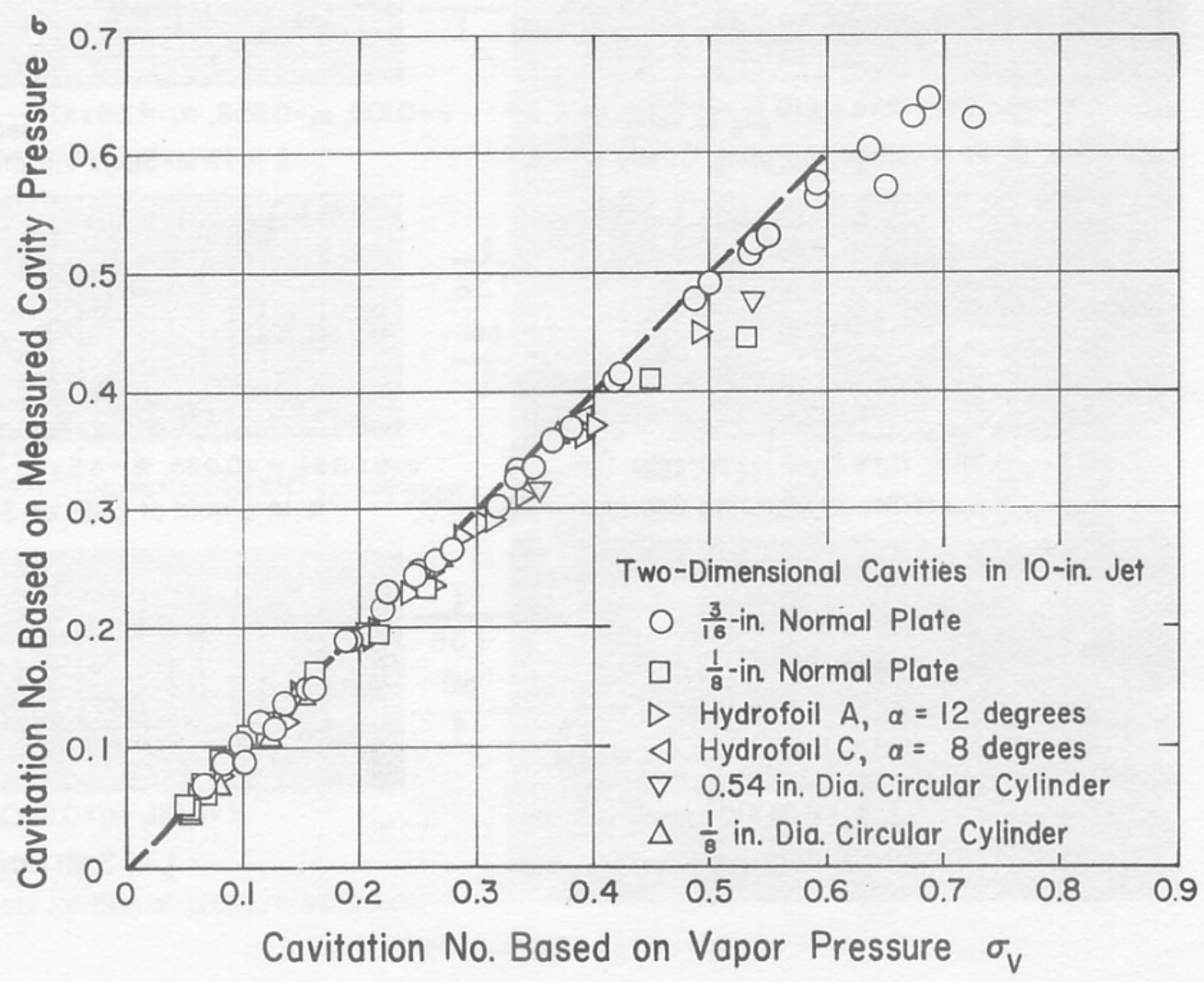

Fig. 4 - Comparison between Cavitation Number Based on Vapor Pressure and Cavitation Number Based on Measured Cavity Pressure 


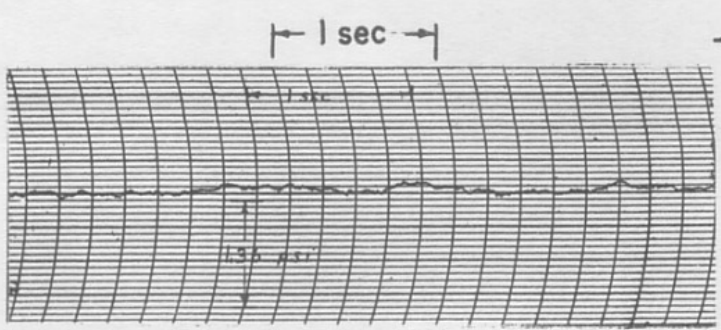

$\sigma_{v}=0.925, W_{A}=0$

a. No Cavity

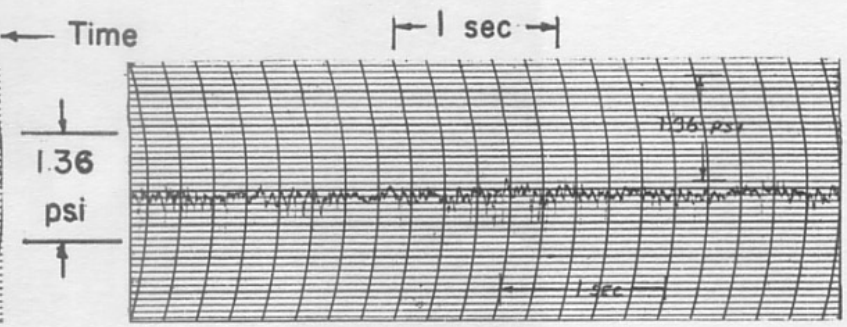

$\sigma=0.251, \sigma_{v}=0.253, W_{A}=0$

b. In Wake of Cavity

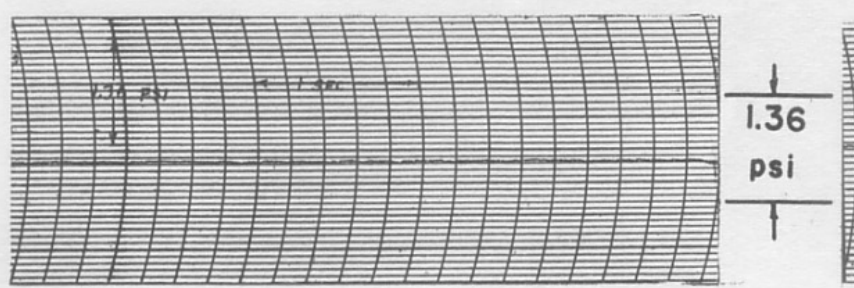

$\sigma=0.0639, \sigma_{\mathrm{V}}=0.0863, W_{A}=0$

c. In Natural Cavity

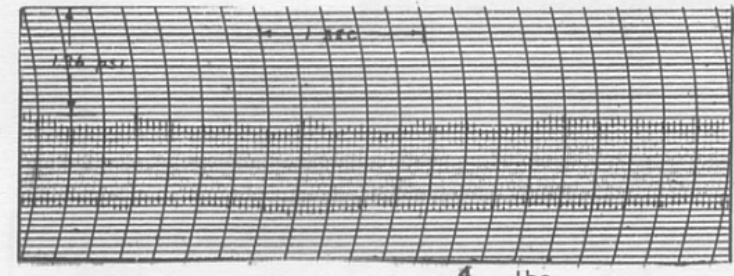

$\sigma=0.0735, \sigma_{V}=0.372, W_{A}=4.6 \times 10^{-4} \frac{\text { lbs }}{\text { sec-in. span }} f=26 \mathrm{cps}$ e. In First-Stage Vibrating Cavity

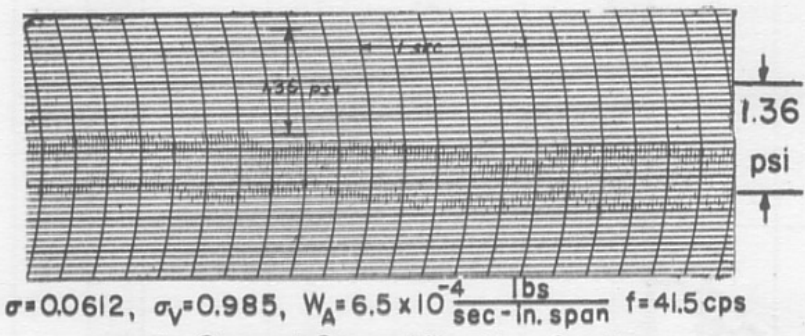

g. In Second-Stage Vibrating Cavity

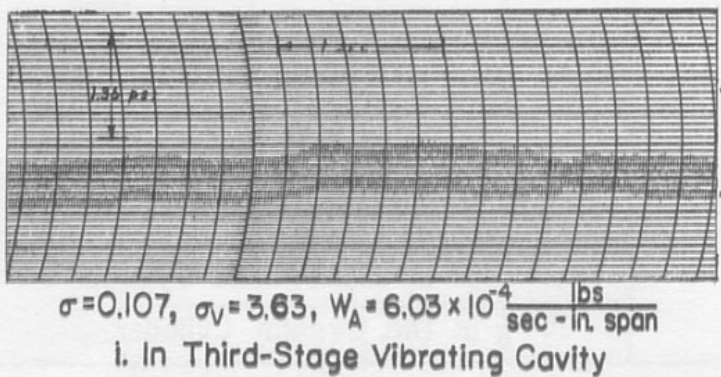

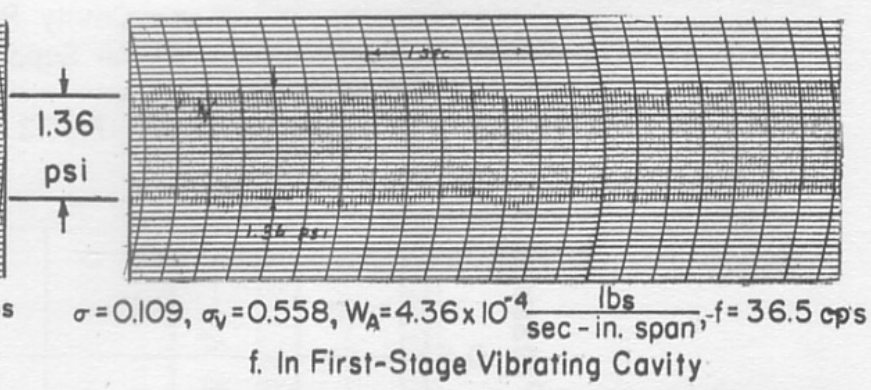

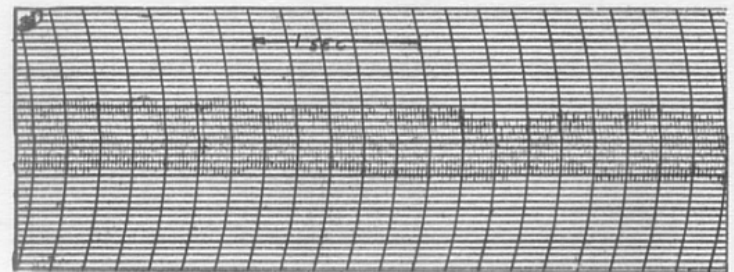

$\sigma=0.0694, \sigma_{V}=0.985, W_{A}=6.5 \times 10^{-4} \frac{\text { lbs }}{\text { sec-in.span }} f=41.5 \mathrm{cps}$

h. In Liquid at Side of Second-Stage
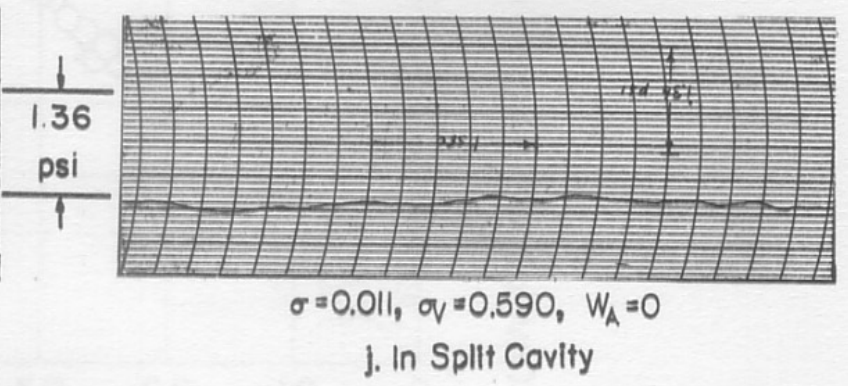

Fig. 5 - Typical Pressure Records

(Two-dimensional, $1 / 8-i n$. normal plate in 10 -in. jet) 


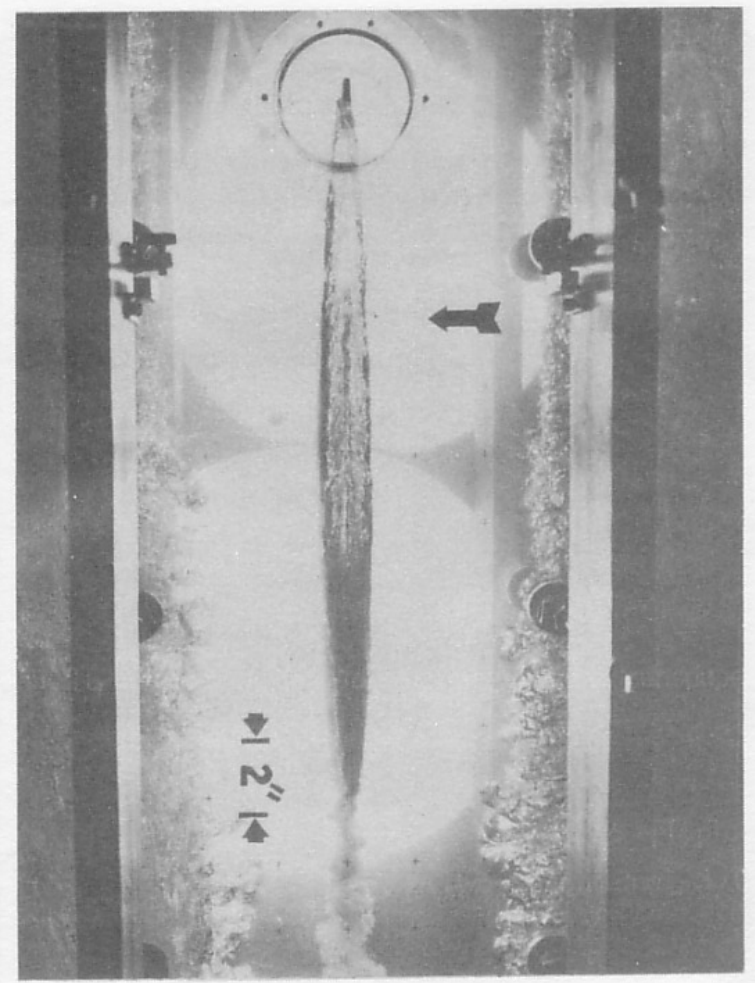
a. Natural Cavity $\sigma=0.0617, \sigma_{V}=0.075$, $\mathrm{V}=45.3 \mathrm{fps}, \mathrm{W}_{\mathrm{A}}=0.00$

b. Ventilated Cavity $\sigma=0.0707, \sigma_{V}=0.171$,
$V=44.0 \mathrm{fps}, W_{A}=0.640 \times 10^{-4} \frac{\text { lbs }}{\text { sec-ln.span }^{-1 n}}$

NOTE : Arrows indicate location of pressure transducer

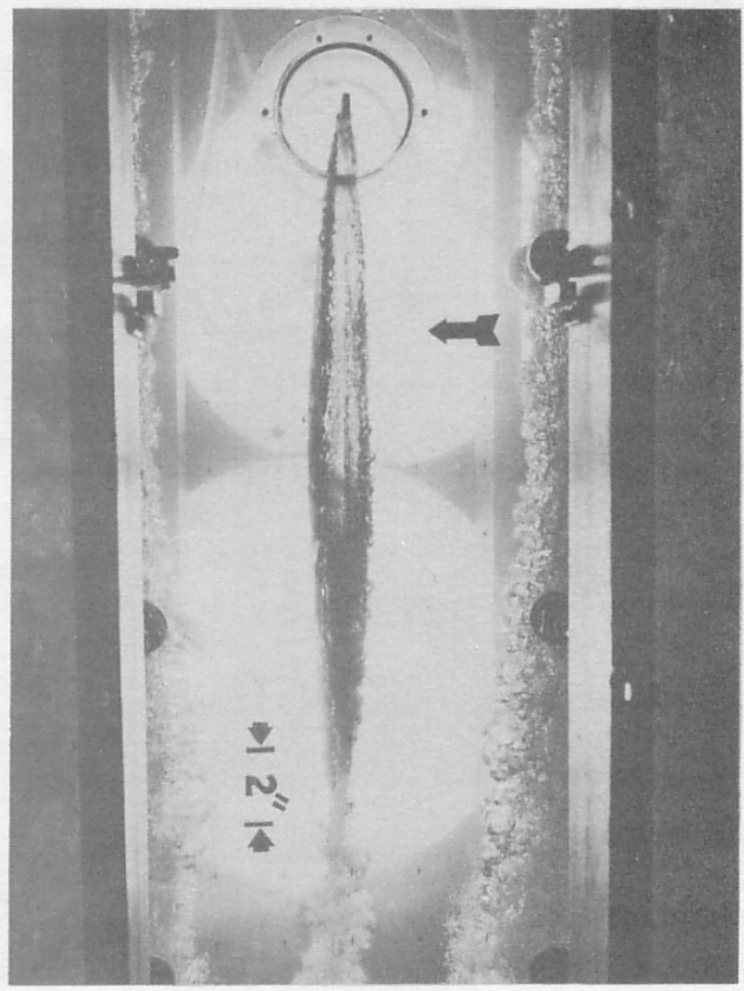

Fig. 6 - Typical Nonvibrating Cavities

(Two-dimensional, 1/8-in. nomal plate in 10-in. jet) 


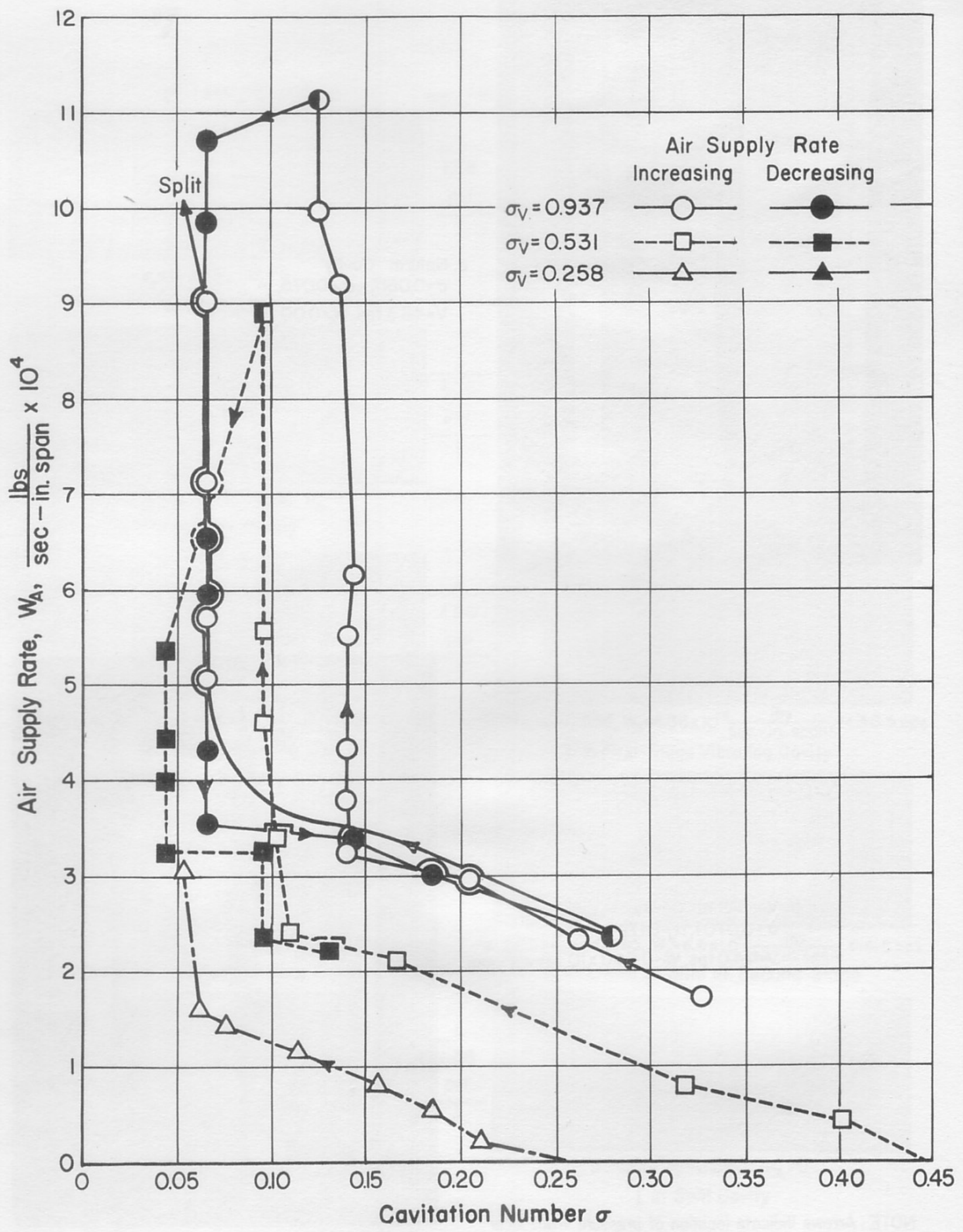

Fig. 7 - Air Supply Rate as a Function of Cavitation Number

(Two-dimensional, 1/8-in. normal plate in 10-in. jet) 


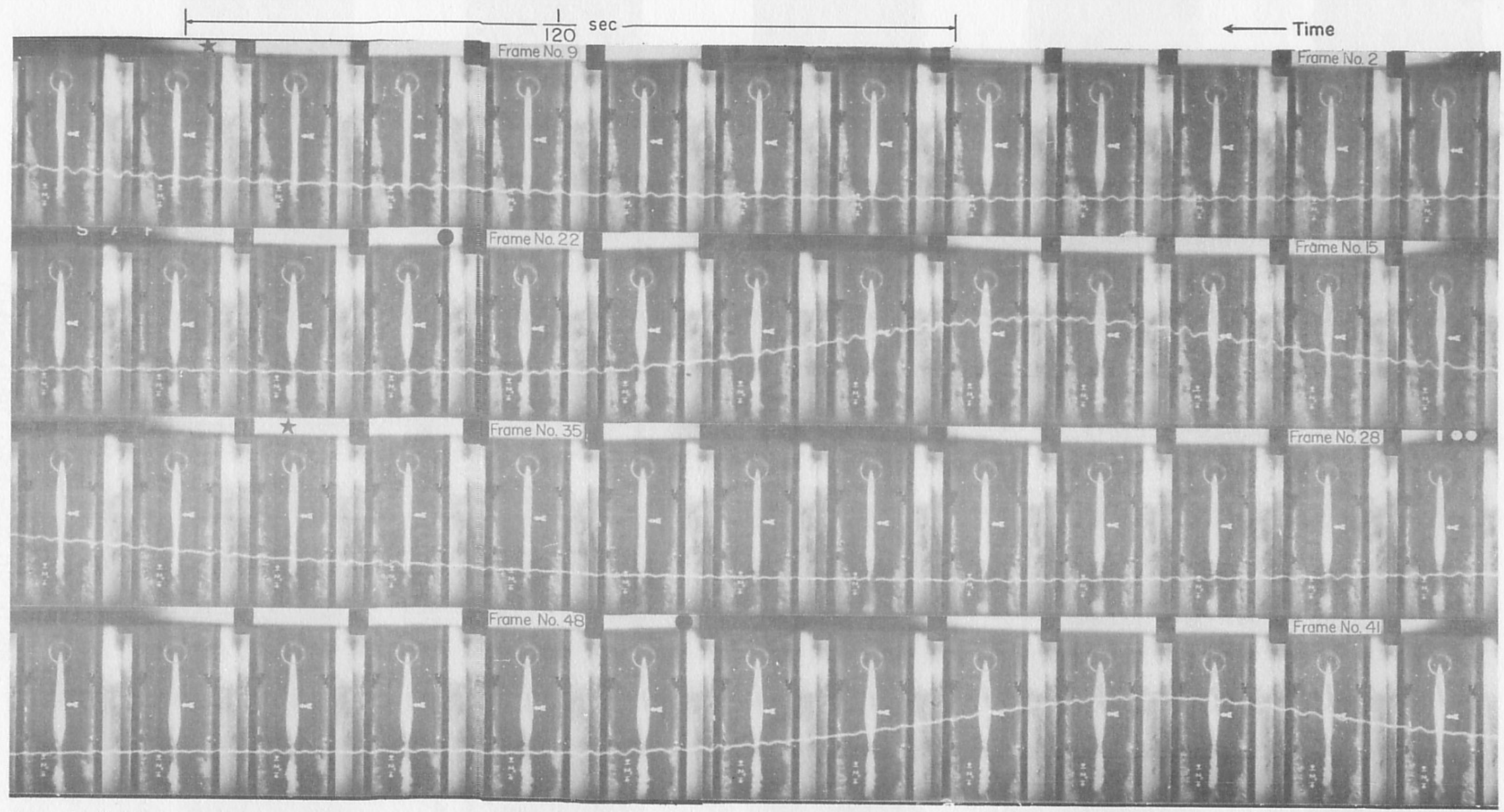

Note: Oscilloscopic pressure trace recorded 5 frames before picture. Pressure positive upward

Fig. 8 - Consecutive Motion Picture Frames for a Vibrating Cavity

(Two-dimensional, 1/8-in. normal plate in 10-in. jet) 

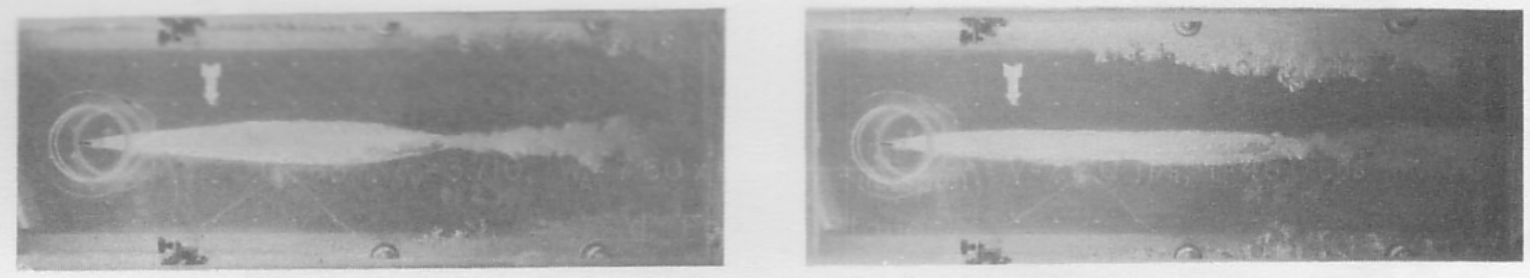

$\sigma=0.0735, \sigma_{v}=0.372, W_{A}=4.60 \times 10^{-4} \frac{\text { lbs }}{\text { sec-in. span }} V=41.5 \mathrm{fps}, f=26.0 \mathrm{cps}$ a. First Stage
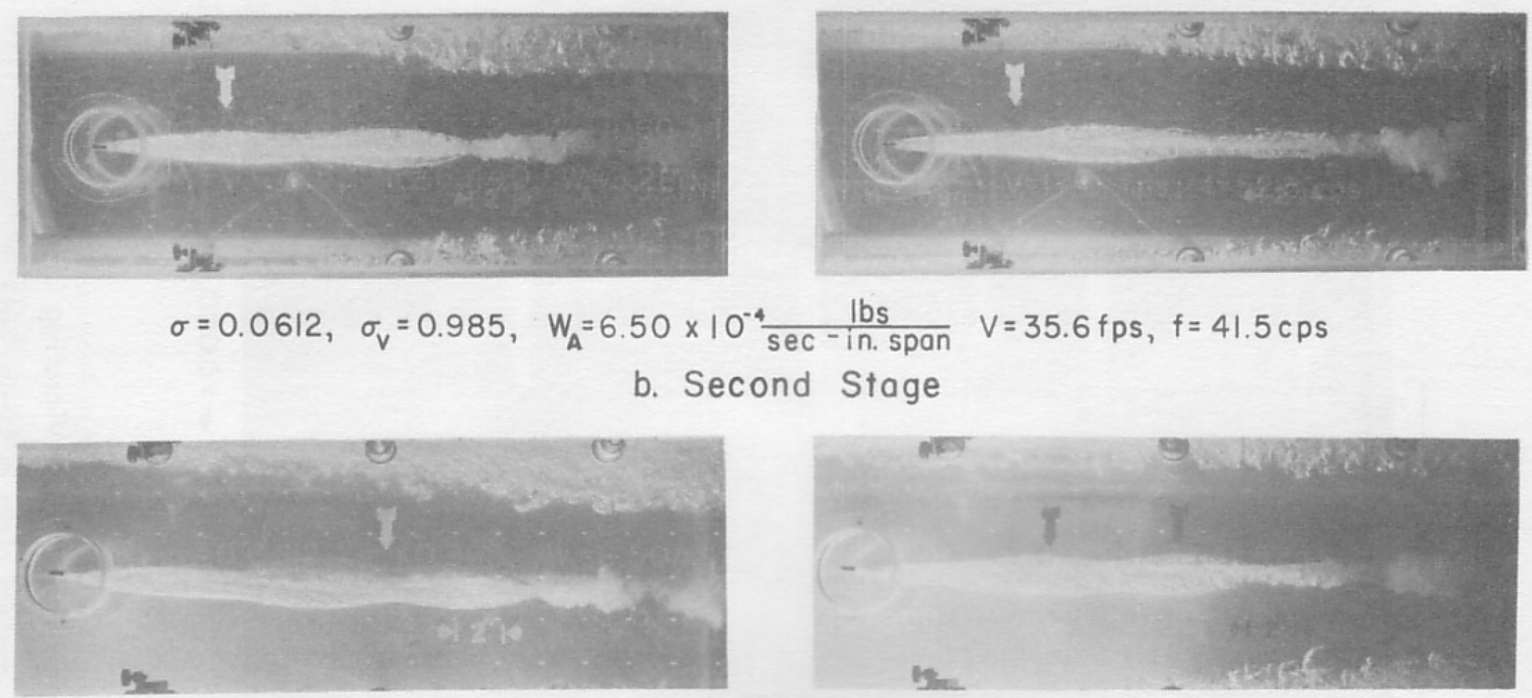

$\sigma=0.0665, \quad \sigma_{v}=2.225, W_{A}=7.40 \times 10^{-4} \frac{\mathrm{lbs}}{\mathrm{sec}-\text { in. span }} \quad V=27.2 \mathrm{fps}, f=49.0 \mathrm{cps}$

c. Third Stage
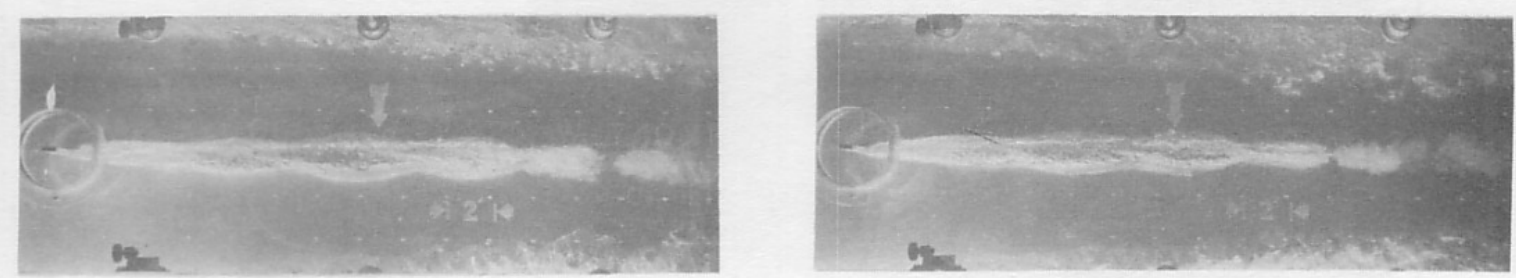

$\sigma=0.0668, \quad \sigma_{v}=3.710, W_{A}=7.60 \times 10^{-4} \frac{\text { lbs }}{\text { sec-in. span }} \quad V=22.0 \mathrm{fps}, f=48.0 \mathrm{cps}$

d. Fourth Stage
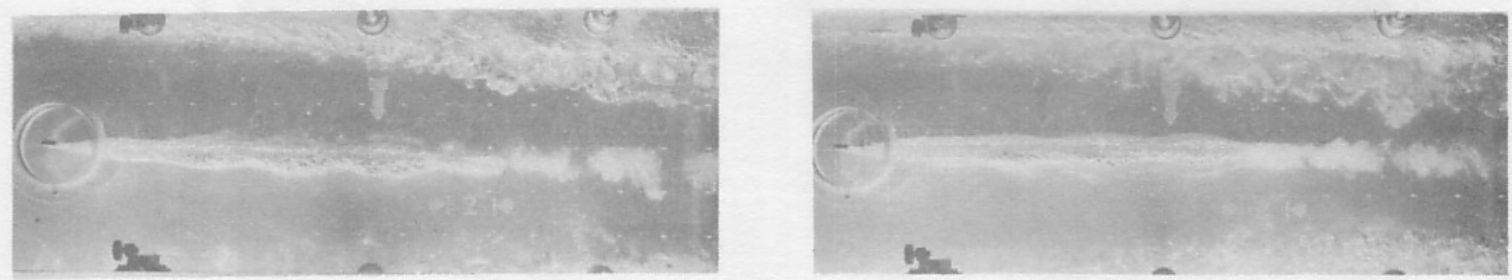

$\sigma=0.0726, \quad \sigma_{v}=6.440, W_{A}=3.28 \times 10^{-4} \frac{\text { lbs }}{\text { sec-in. span }} V=17.0 \mathrm{fps}, f=52.5 \mathrm{cps}$

e. Fifth Stage

Fig. 9 - Several Vibrating Cavities for a Two-Dimensional Normal Plate (1/8-in. plate, 10-in. jet) 

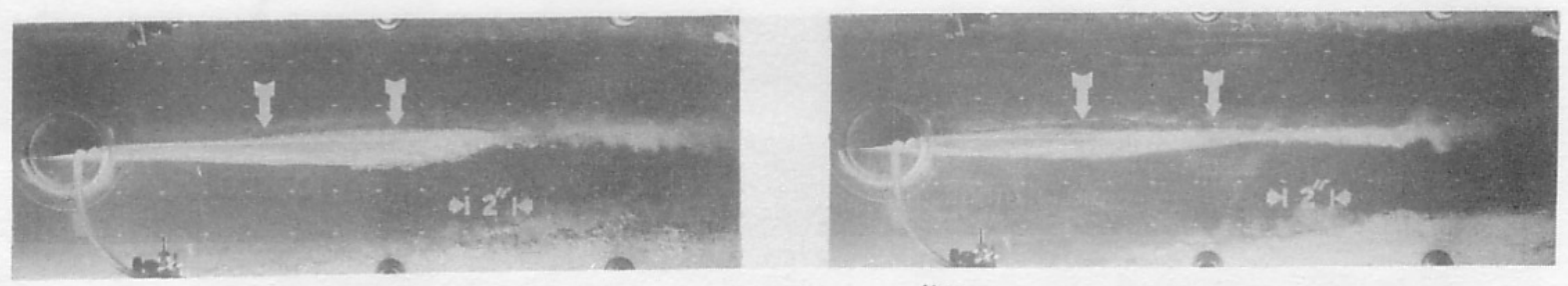

$\sigma=0.0544, \sigma_{v}=0.270, W_{A}=2.36 \times 10^{-4} \frac{\text { lbs }}{\text { sec-in. span }} \quad V=42.5 \mathrm{fps}, f=26.8 \mathrm{cps}$

a. First Stage
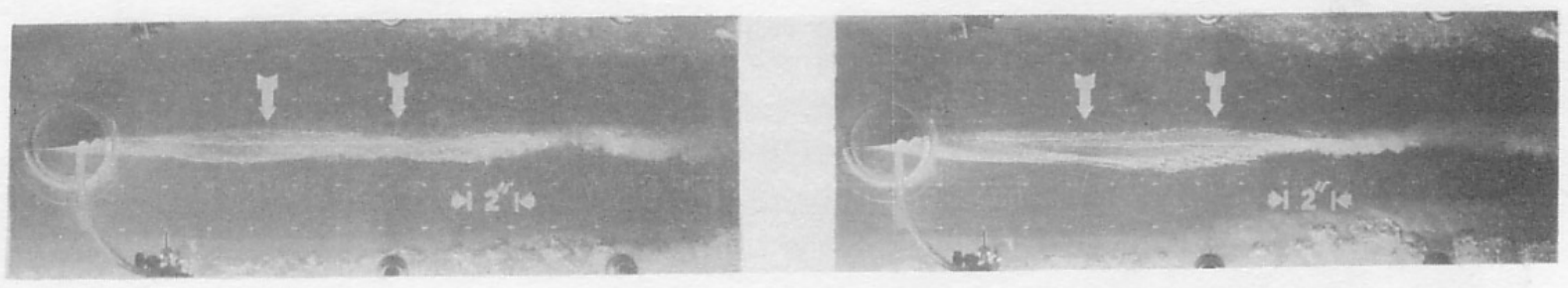

$$
\begin{gathered}
\sigma=0.0556, \sigma_{v}=0.869, W_{A}=6.45 \times 10^{-4} \frac{\text { lbs }}{\sec -\text { in. span }} \quad V=35.5 \mathrm{fps}, f=40.7 \mathrm{cps} \\
\text { b. Second Stage }
\end{gathered}
$$
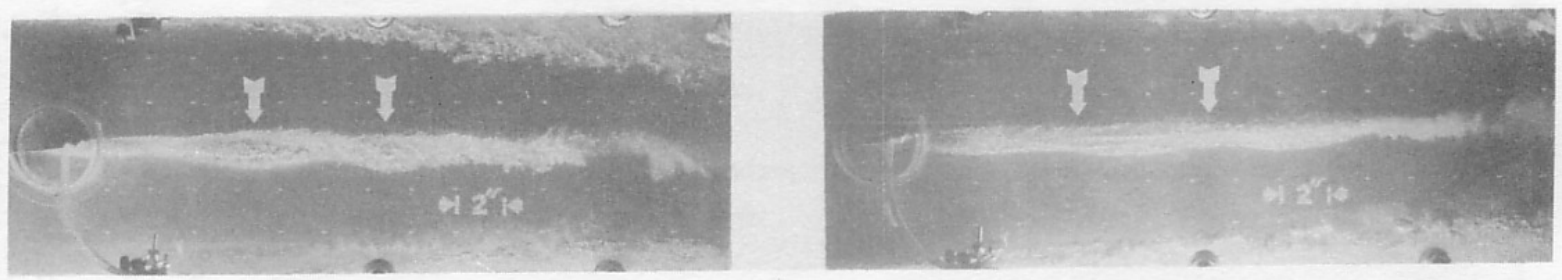

$$
\begin{gathered}
\sigma=0.0671, \sigma_{V}=1.960, W_{A}=7.04 \times 10^{-4} \frac{1 \text { Ds }}{\text { sec-in. span }} V=28.9 \mathrm{fps}, f=46.0 \mathrm{cps} \\
\text { C. Third Stage }
\end{gathered}
$$
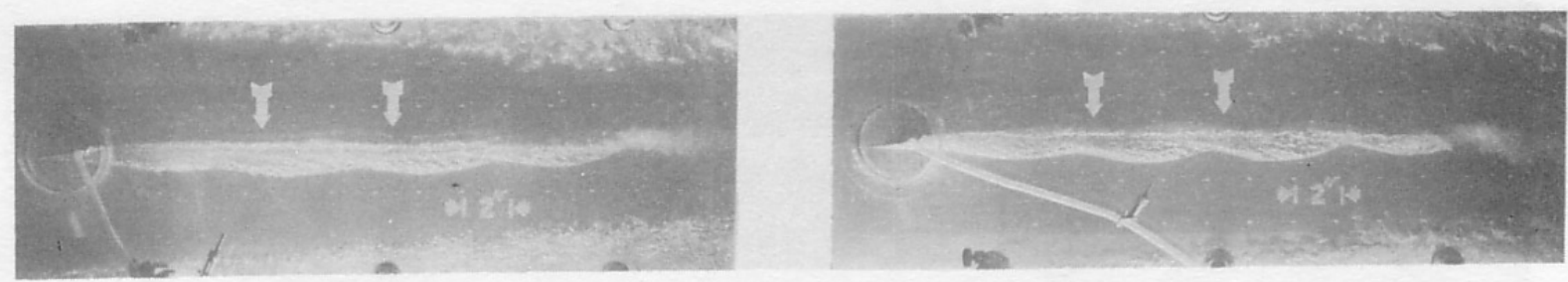

$\sigma=0.0601, \sigma_{v}=3.050, W_{A}=3.83 \times 10^{-4} \frac{\text { lbs }}{\text { sec-in. span }} \quad V=24.2 \mathrm{fps}, f=46.0 \mathrm{cps}$

\section{d. Fourth Stage}
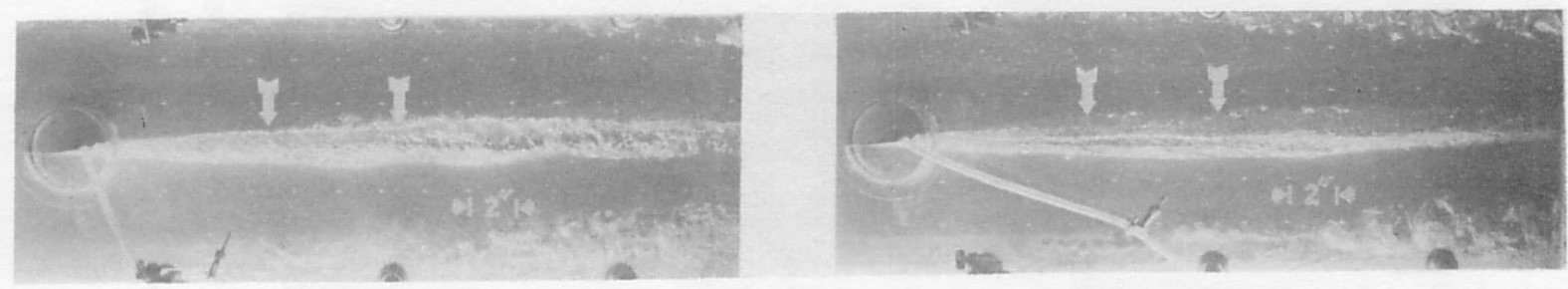

$\sigma=0.0252, \sigma_{v}=3.110, W_{A}=3.96 \times 10^{-4} \frac{\text { lbs }}{\text { sec-in. span }} \quad V=23.6 \mathrm{fps}, f=45.0 \mathrm{cps}$

e. Fifth Stage

Fig. 10 - Several Vibrating Cavities for a Two-Dimensional Hydrofoil

(Hydrofoil A, $a=8$ degrees, 10-in. jet) 


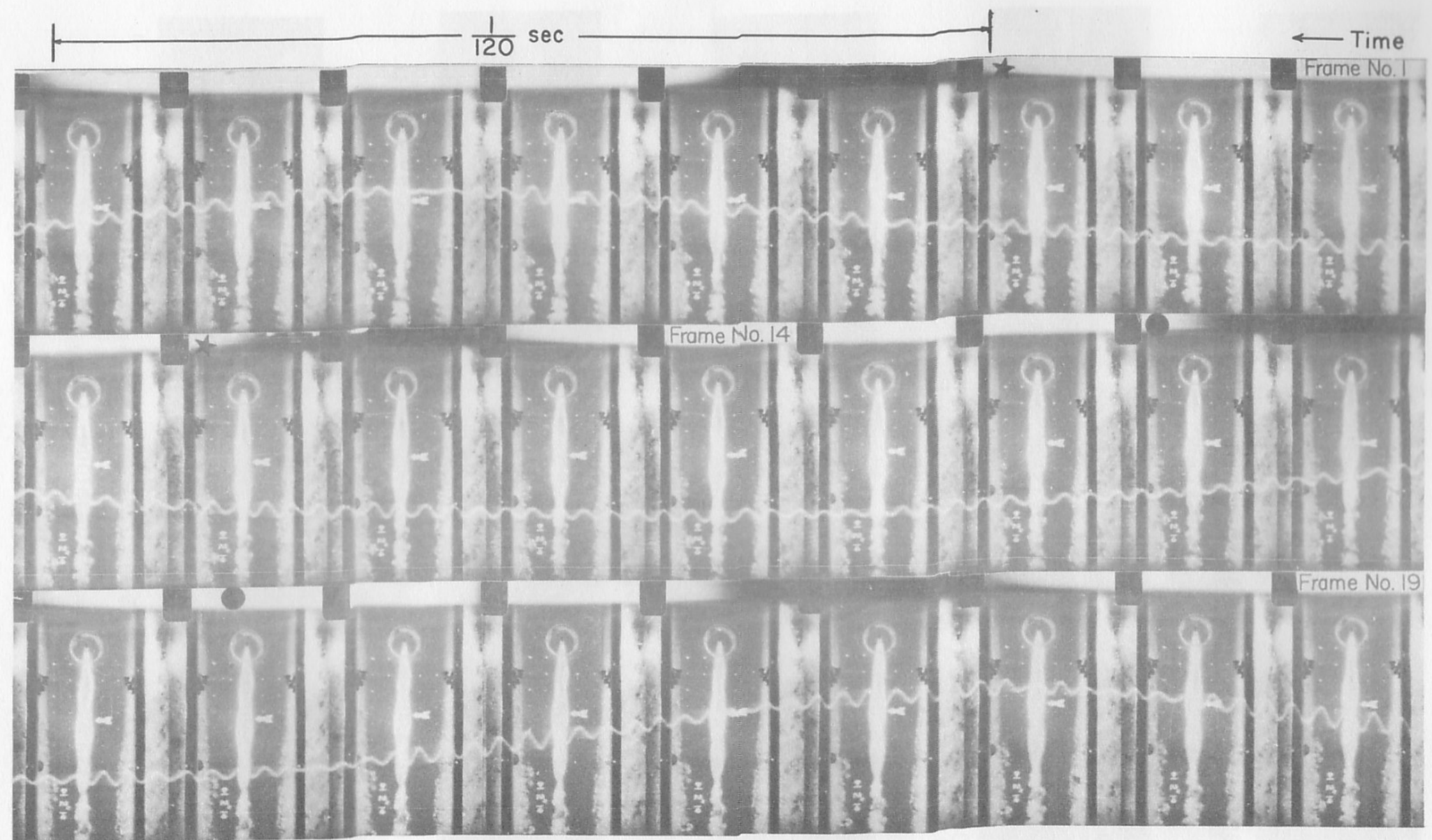

Note: Oscilloscopic pressure trace recorded 5 frames before picture.

Pressure positive upward

$\sigma=0.103, \quad \sigma_{v}=3.61, \quad W_{A}=5.79 \times 10^{-4} \frac{\text { lbs }}{\text { sec-in.span }} \quad V=21.85 \mathrm{fps}$

Fig. 11 - Consecutive Motion Picture Frames for a Three-Stage Cavity

(Two-dimensional, 1/8-in. normal plate in 10-in. jet) 

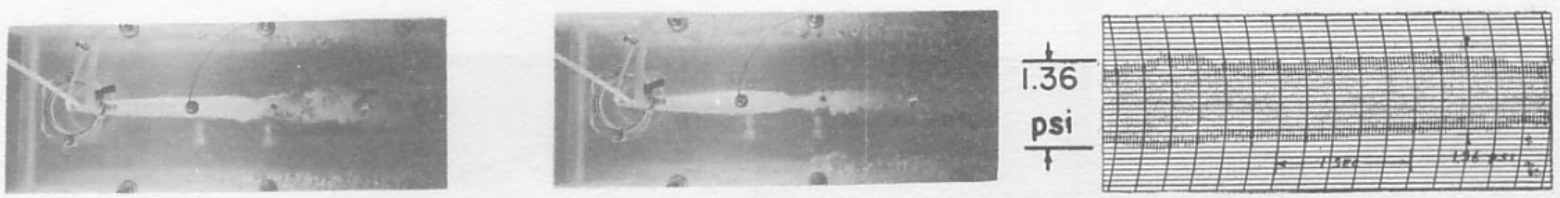

$\sigma=0.0829, \sigma_{V}=0.481, W_{A}=2.5 \times 10^{-4} \frac{\mathrm{lbS}}{\mathrm{sec}-\text { in. span }}, V=38.5 \mathrm{fps}, f=40.5 \mathrm{cps}$

a. First Stage
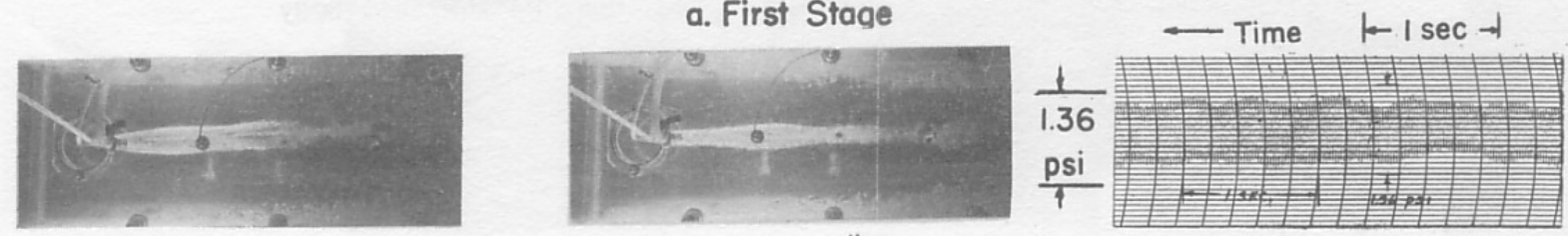

$$
\sigma=0.0586, \sigma_{V}=0.922, W_{A}=3.68 \times 10^{-4} \frac{\text { lbs }}{\text { sec-in. span }}, V=34.7 \mathrm{fps}, f=49.0 \mathrm{cps}
$$

b. Second Stage
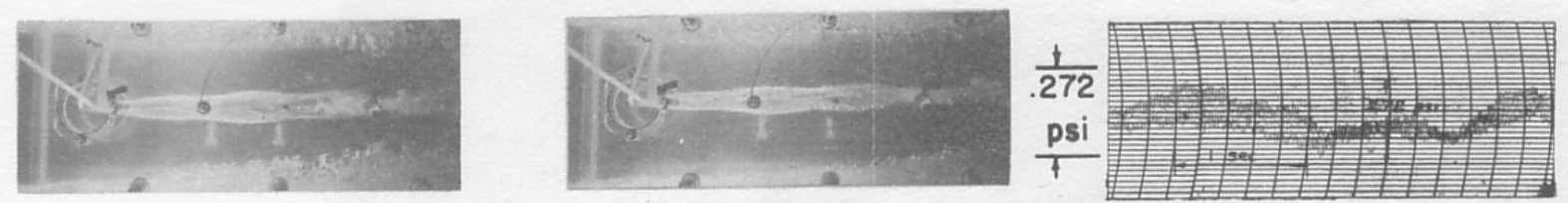

$\sigma=0.0556, \sigma_{V}=2.02, W_{A}=4.64 \times 10^{-4} \frac{\mathrm{lbs}}{\text { sec-in. span }}, V=27.5 \mathrm{fps}, f=50.0 \mathrm{cps}$

\section{c. Third Stage}

Fig. 12 - Vibrating Cavities for a Half-Span Normal Plate

$$
\text { (1/8-in. plate, 10-in. jet) }
$$
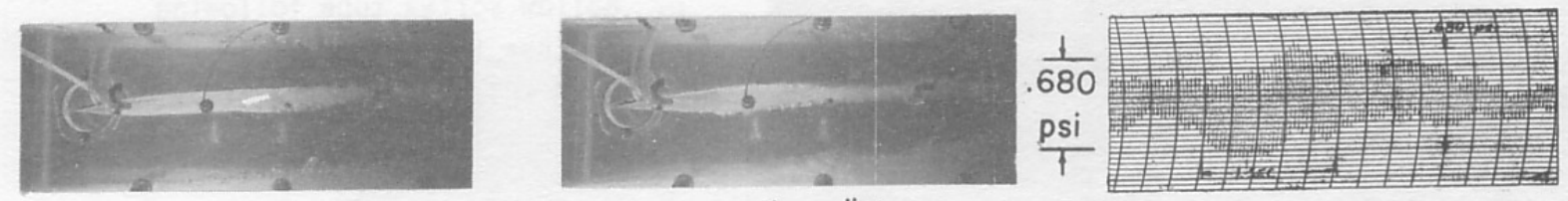

$\sigma=0.0598, \sigma_{V}=0.364, W_{A}=2.46 \times 10^{-4} \frac{\text { lbs }}{\text { sec-in. spon }}, V=41.4 \mathrm{fps}, f=30.9 \mathrm{cps}$

\section{a. First Stage}
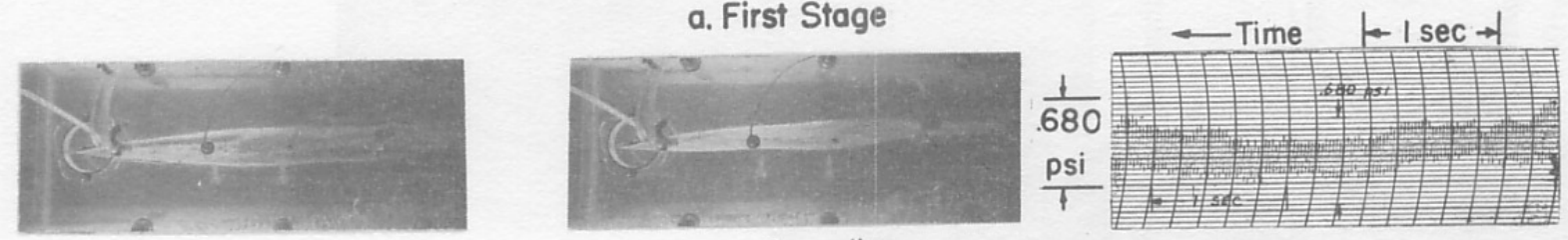

$\sigma=0.0412, \sigma_{V}=0.610, W_{A}=4.27 \times 10^{-4} \frac{\text { lbs }}{\text { sec-in. span }}, V=38.1 \mathrm{fps}, f=38.2 \mathrm{cps}$

\section{b. Second Stage}
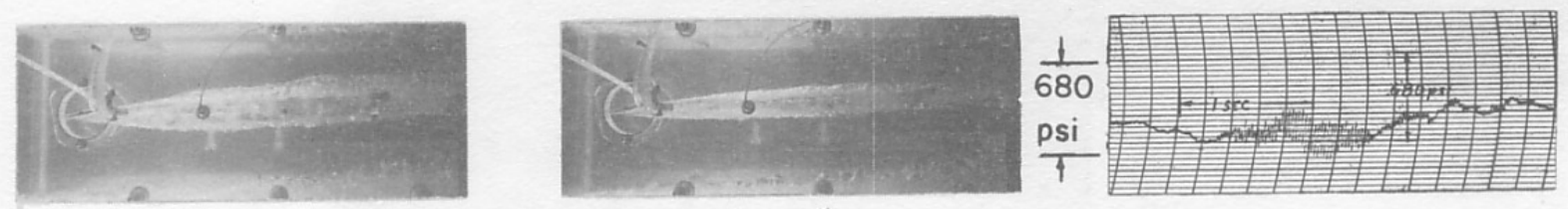

$$
\begin{gathered}
\sigma=0.0255, \sigma_{V}=1.11, W_{A}=5.41 \times 10^{-4} \frac{\text { lbs }}{\text { sec-in.span }}, V=33.2 \mathrm{fps}, f=42.0 \mathrm{cps} \\
\text { c. Third Stage }
\end{gathered}
$$

Fig. 13 - Vibrating Cavities for a Half-Span Hydrofoil (Hydrofoil A, 12 degree angle of attack in 10-in. jet) 


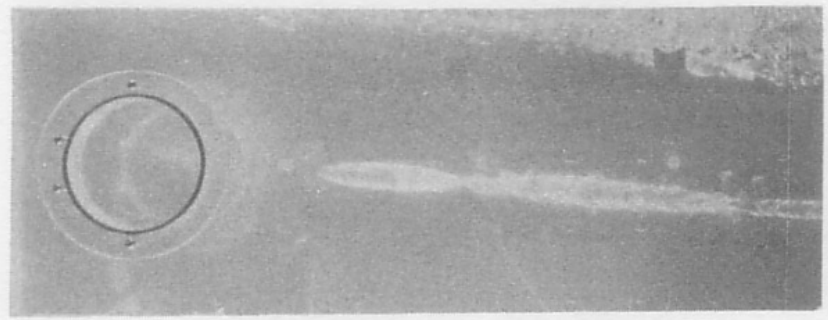

a. Hollow vortex tube has not reached the test body

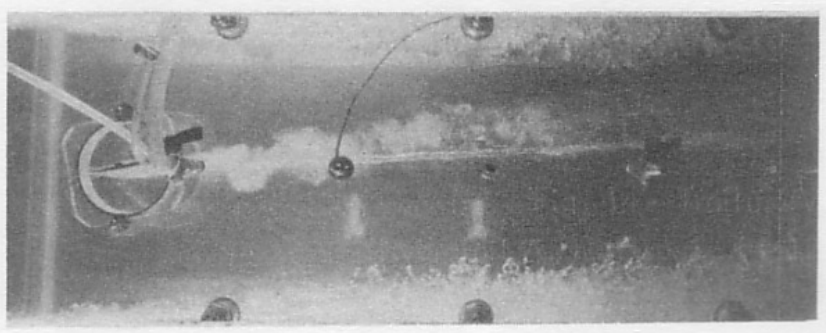

b. Hollow vortex tube just after reaching the test body

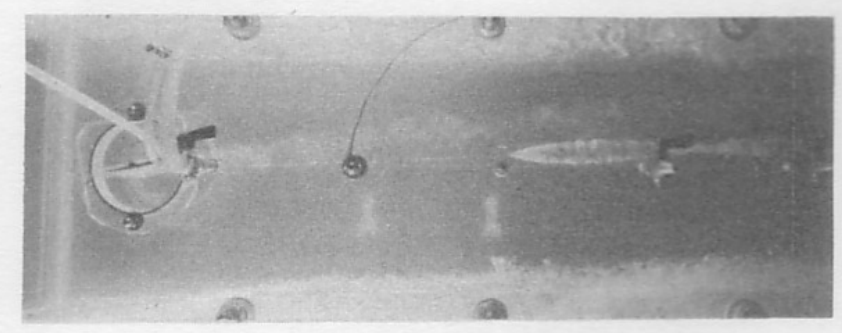

c. Hollow vortex tube following another tube of the same type which was just emptied

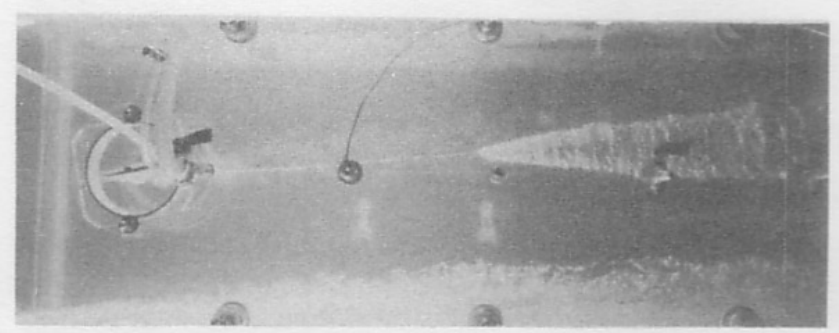

d. Split trailing vortex tube

Fig. 14 - Trailing Vortex Tubes

(Hydrofoil A, half-span, $a=12$ degrees, 10-in. jet) 


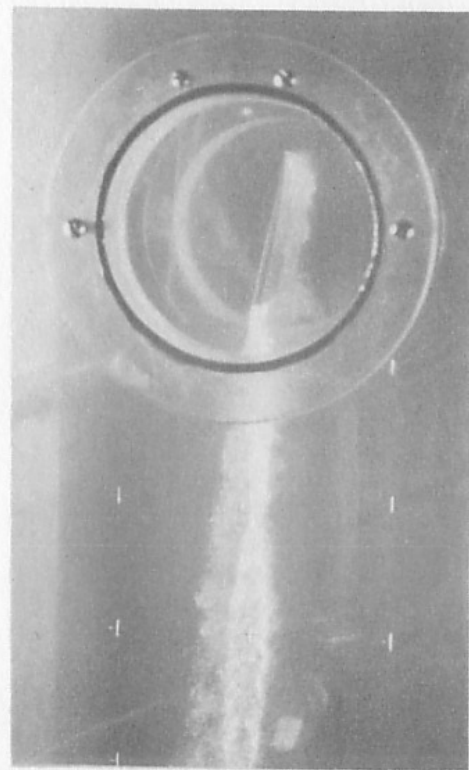

Vortex tube not ventilating test body

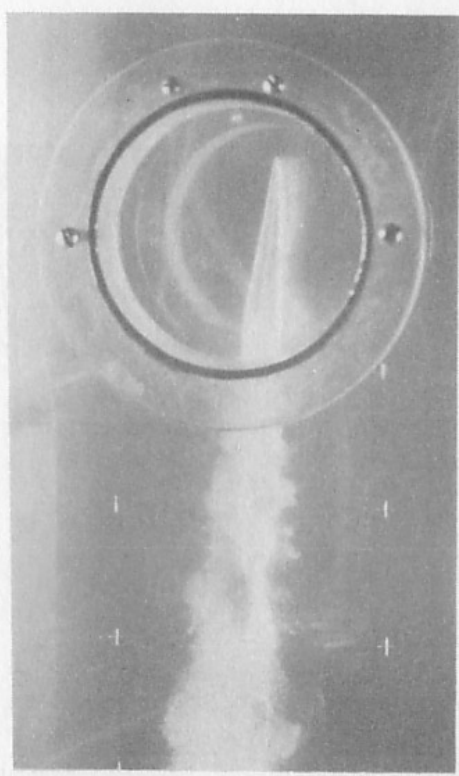

Vortex tube ventilating end, only, of test body

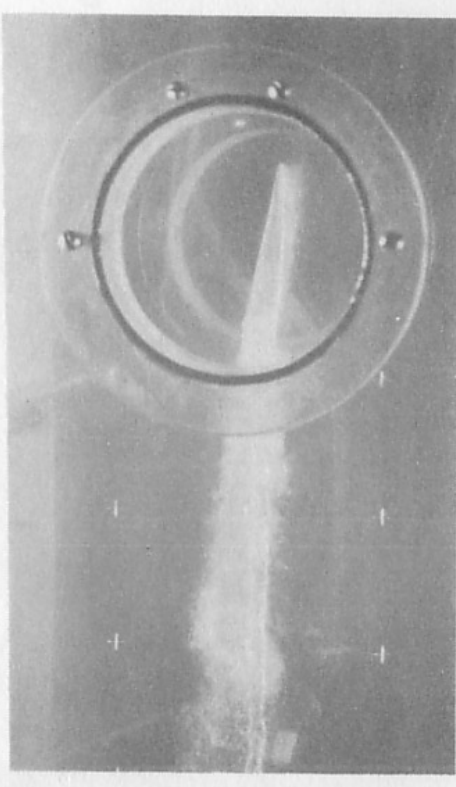

Vortex tube ventilating end, only, of test body

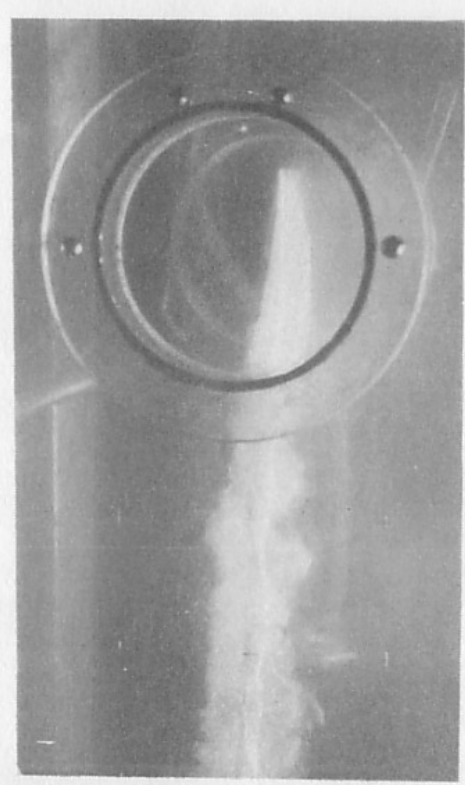

Vortex fube ventilating entire span of test body a. $W_{A}=0.204 \times 10^{-4} \frac{\mathrm{lbs}}{\text { sec-in. span }}$ $\sigma_{v}=1.28, V=32.8 \mathrm{fps}$ b. $\begin{aligned} W_{A} & =0.783 \times 10^{-4} \frac{\mathrm{lbs}}{\mathrm{sec}-\text { in. span }} \\ \sigma_{v} & =1.28, \quad V=32.8 \mathrm{fps}\end{aligned}$

Fig. 15 - Ventilation through Trailing Vortex Tubes

(Hydrofoil A, half-span, $\alpha=12$ degrees, 10-in. jet) 

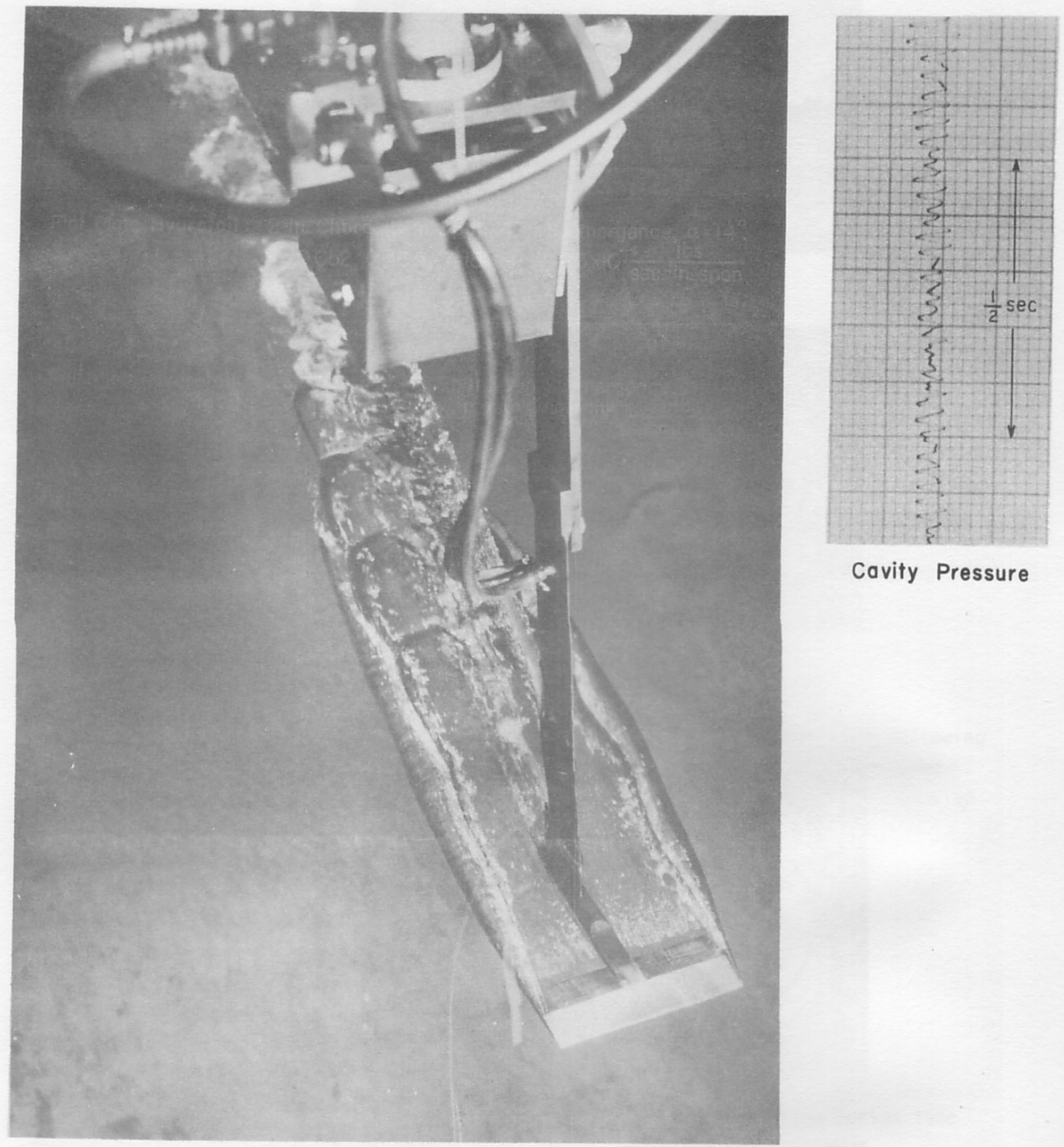

Flat Plate Hydrofoil : 2-in. Chord, 5-in. Span, 4-in. Submergence, $\alpha=14^{\circ}$, $\sigma=0.052, \sigma_{V}=6.5, V=18 \mathrm{fps}, W_{A}=7.5 \times 10^{-4} \frac{\mathrm{lbs}}{\mathrm{sec}^{-i n} \text { span }}$

Fig. 16 - Ventilated Cavity in a Towing Tank 

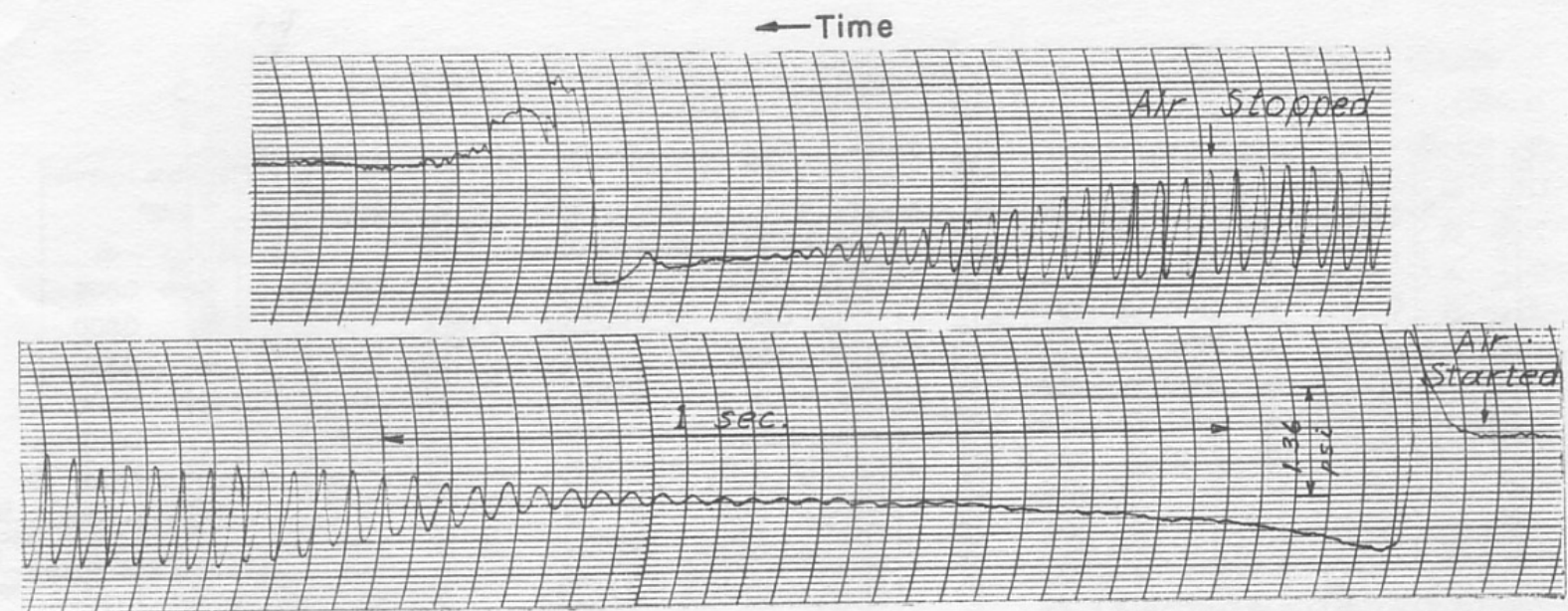

First-Stage Vibrating Cavity, $\sigma_{c}=0.0856, \sigma_{v}=0.470,0 \leq W_{A} \geq 3.62 \times 10^{-3} \mathrm{lbs} / \mathrm{sec}-$ in. span-in. width

Fig. 17 - Pressure Record during Change in Air Supply Rate 

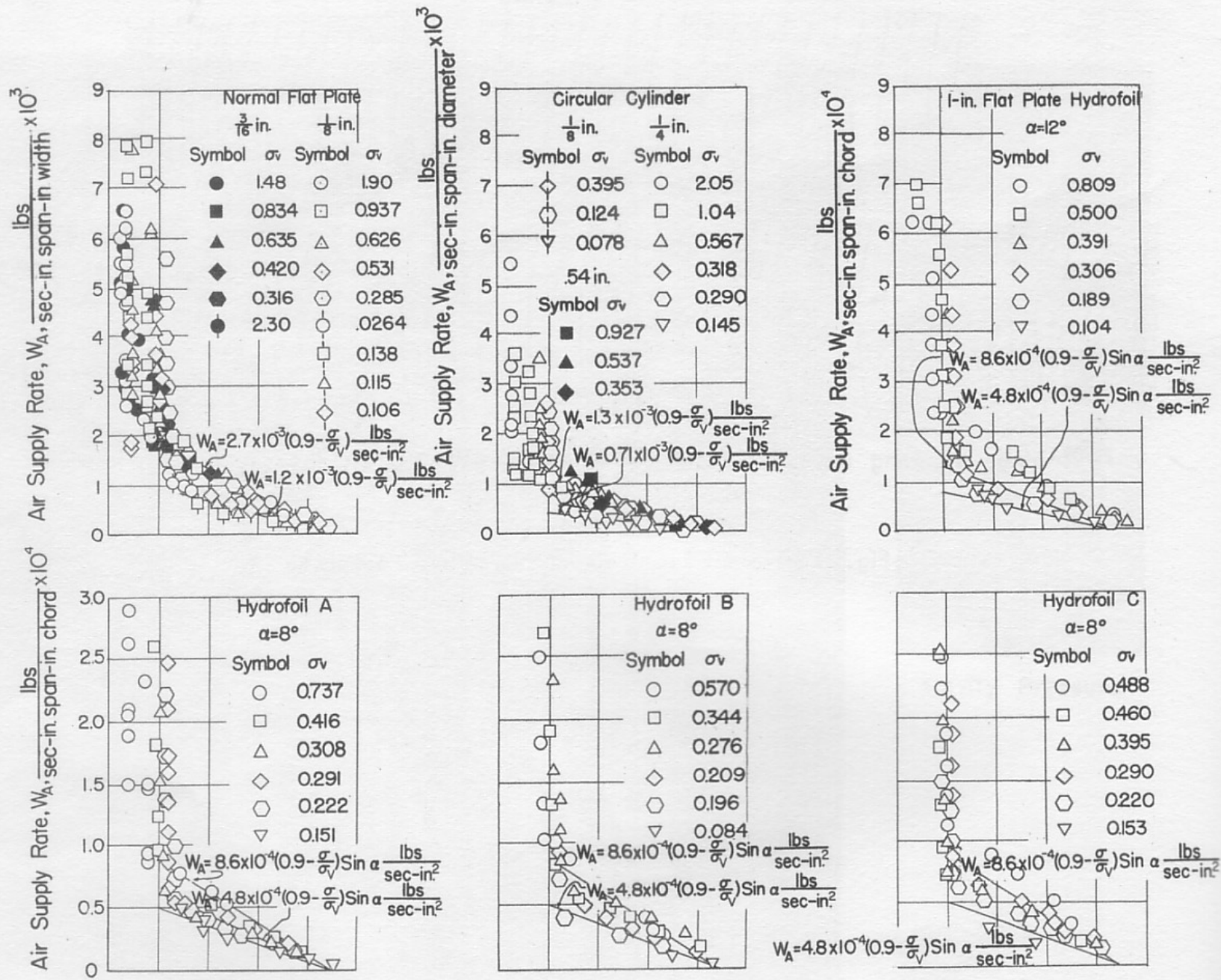

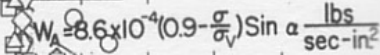

$\frac{\mathrm{lbs}}{\mathrm{sec}-\mathrm{in}^{2}}$

lbs

$W_{A}=4.8 \times 10^{-4}\left(0.9-\frac{\sigma}{\sigma_{V}}\right) \sin a \frac{7 \mathrm{bs}}{\sec ^{2} \text {-in }^{2}}$
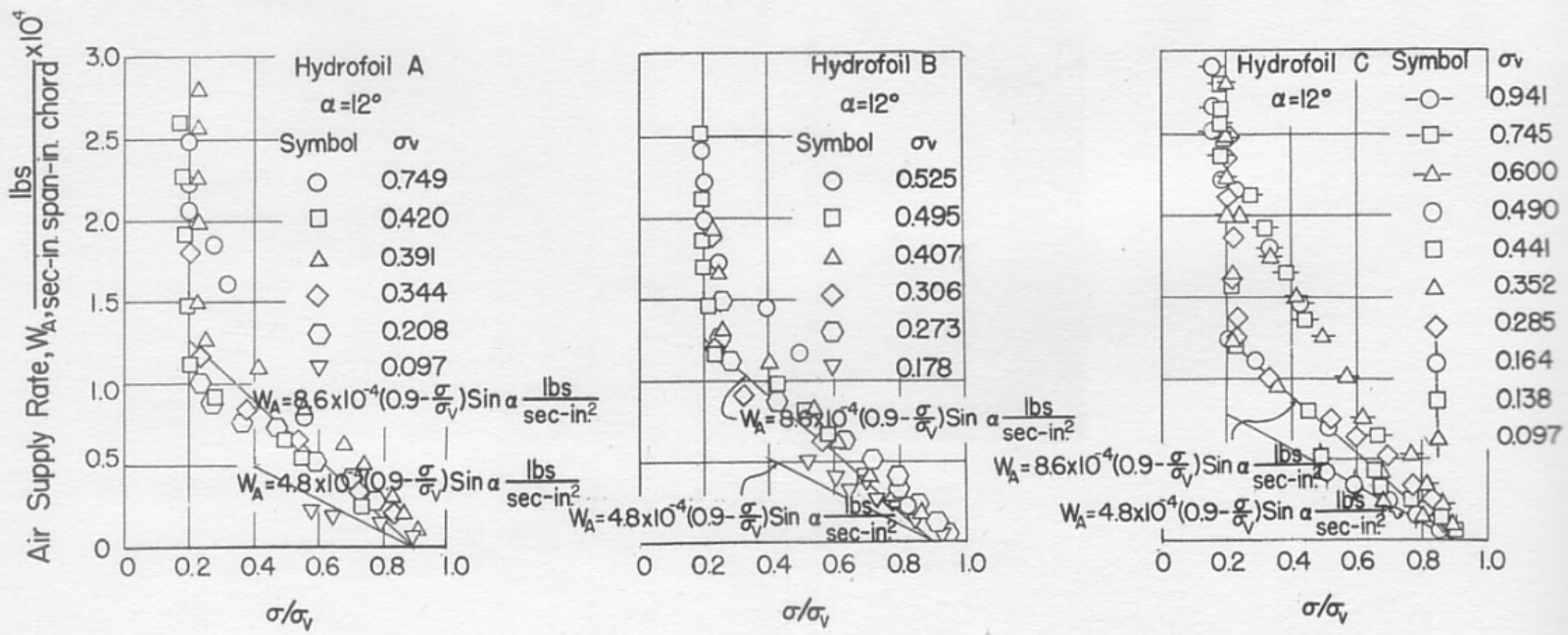

Fig. 18 - Air Supply Rate for Two-Dimensional Bodies 

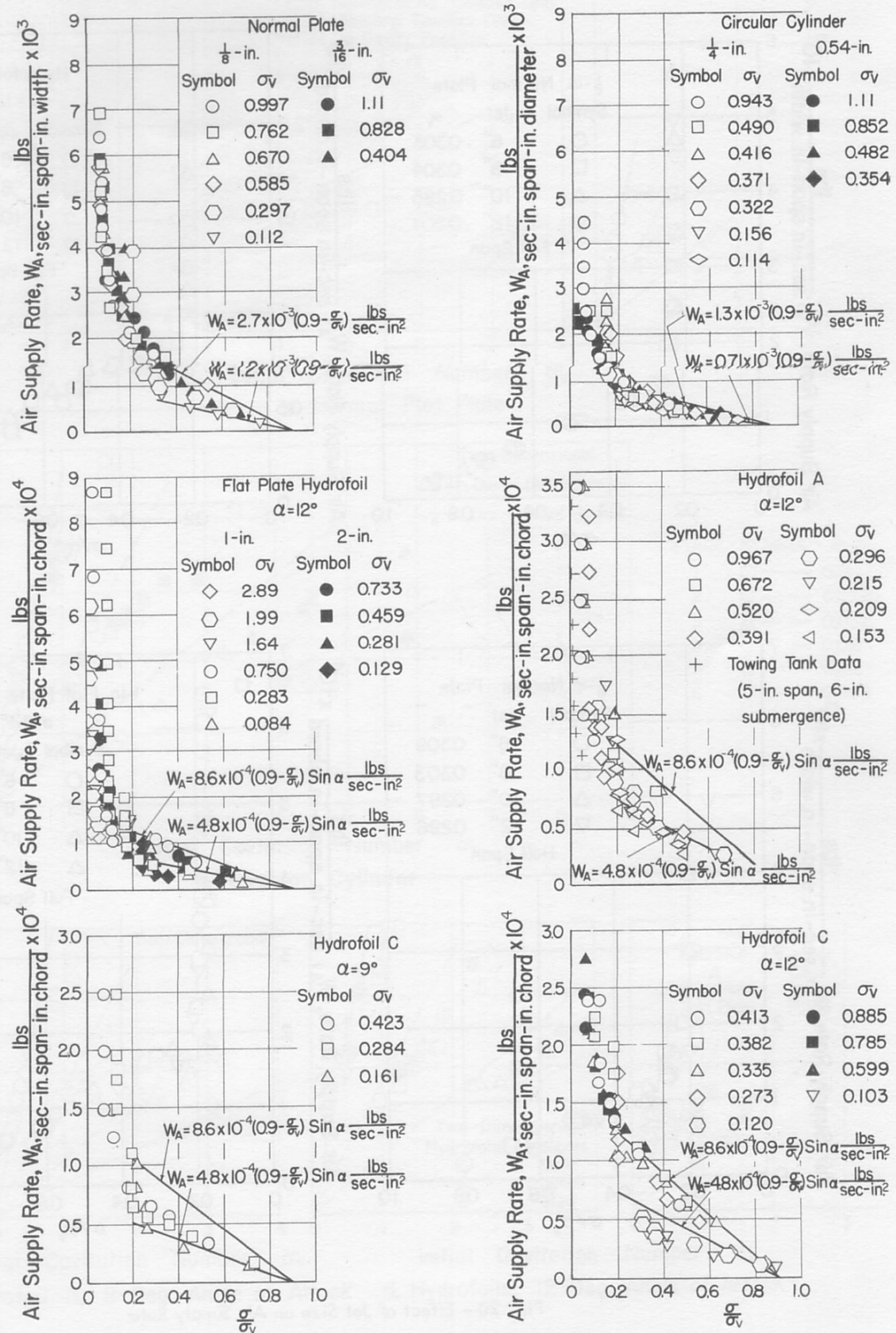

Fig. 19 - Air Supply Rate for Half-Span Bodies 

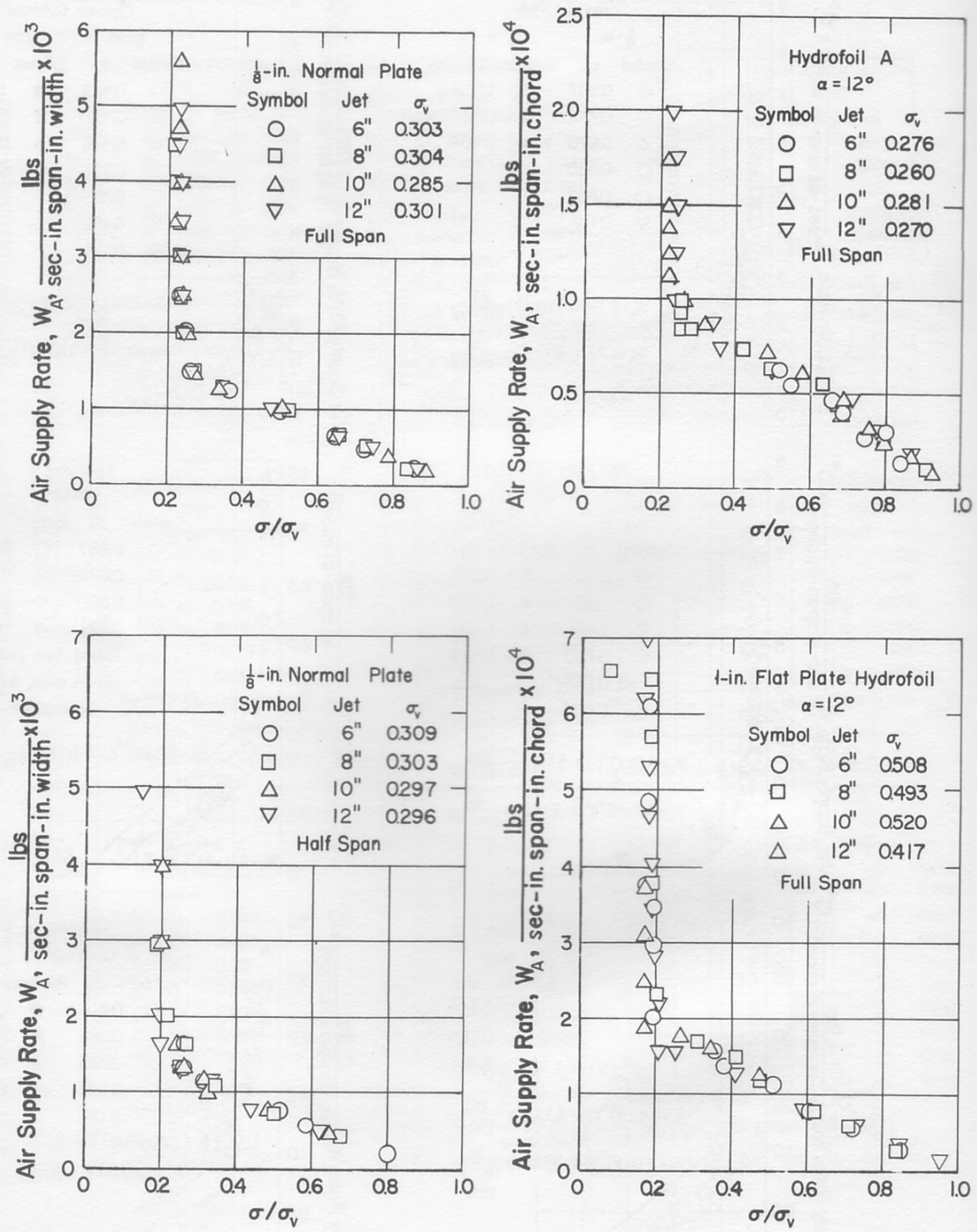

Fig. 20 - Effect of Jet Size on Air Supply Rate 

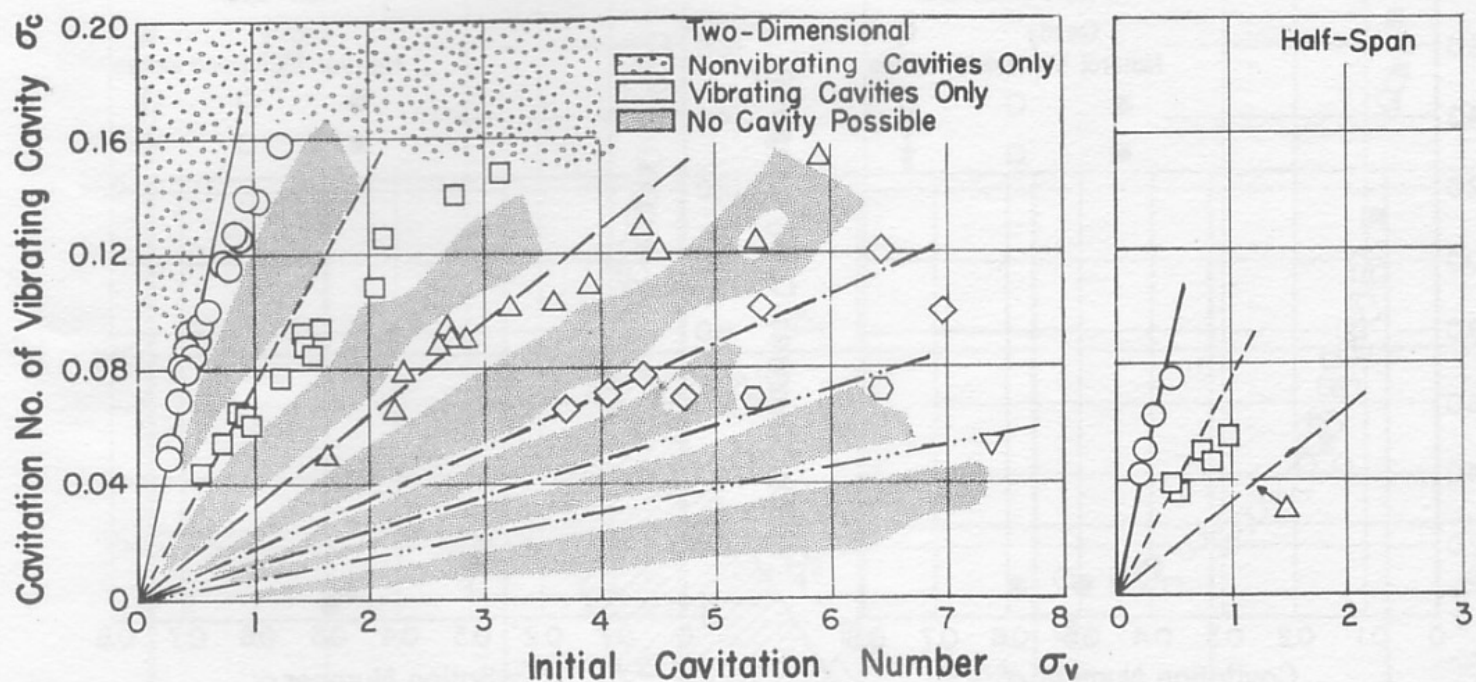

a. Normal Flat Plate

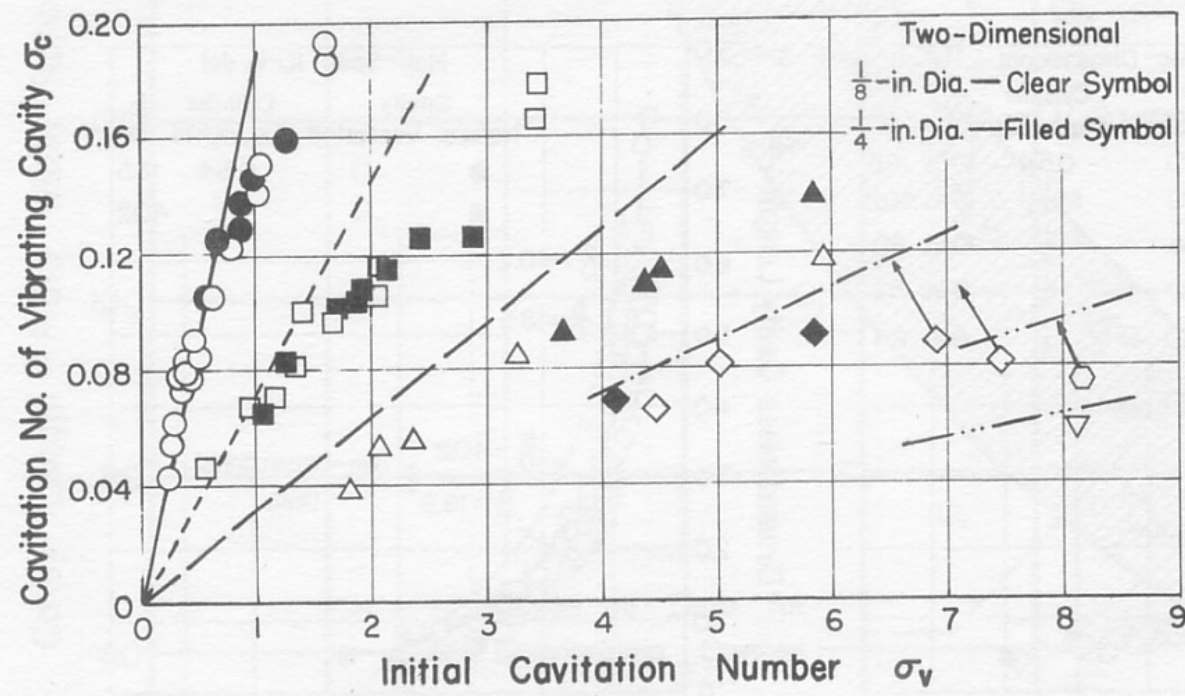

STAGE- SYMBOL $-\sigma_{c} / \sigma_{V}$

I $\mathrm{O} \longrightarrow 0.19$

2 口---- 0.0725

$3 \quad \Delta--0.032$

$4 \Delta-.0 .018$

$50-\cdots-0.012$

$6 \quad \nabla-\cdots-0.008$

6

b. Circular Cylinder

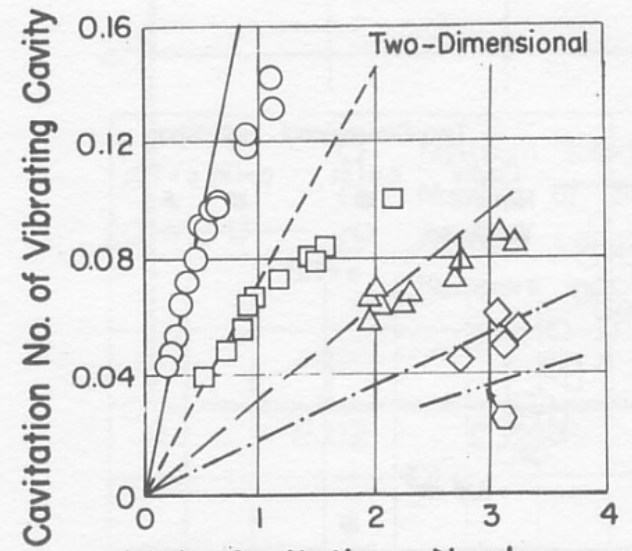

Initial Cavitation Nưmber $\sigma_{v}$

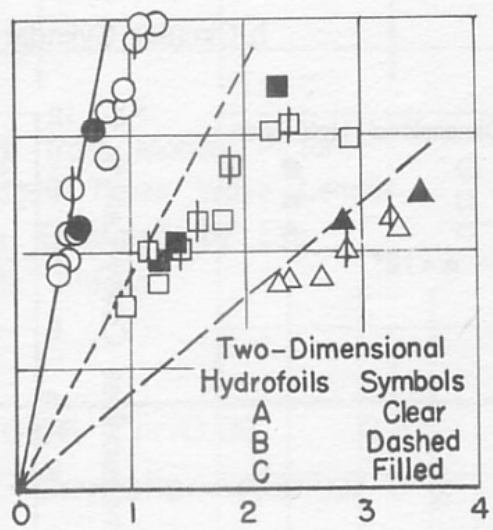

Initial Cavitation Number $\sigma_{v}$

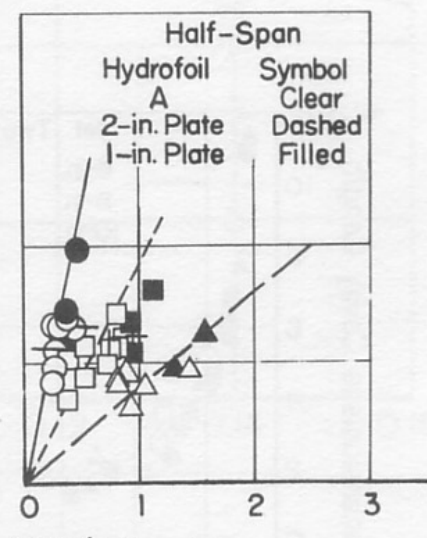
d. Hydrofoils, 12-Deg. Angle of Attack
c. Hydrofoil A, 8-Deg. Angle of Attack

Fig. 21 - Cavitation Number for Each Vibrating Stage as a Function of Initial Cavitation Number 
44
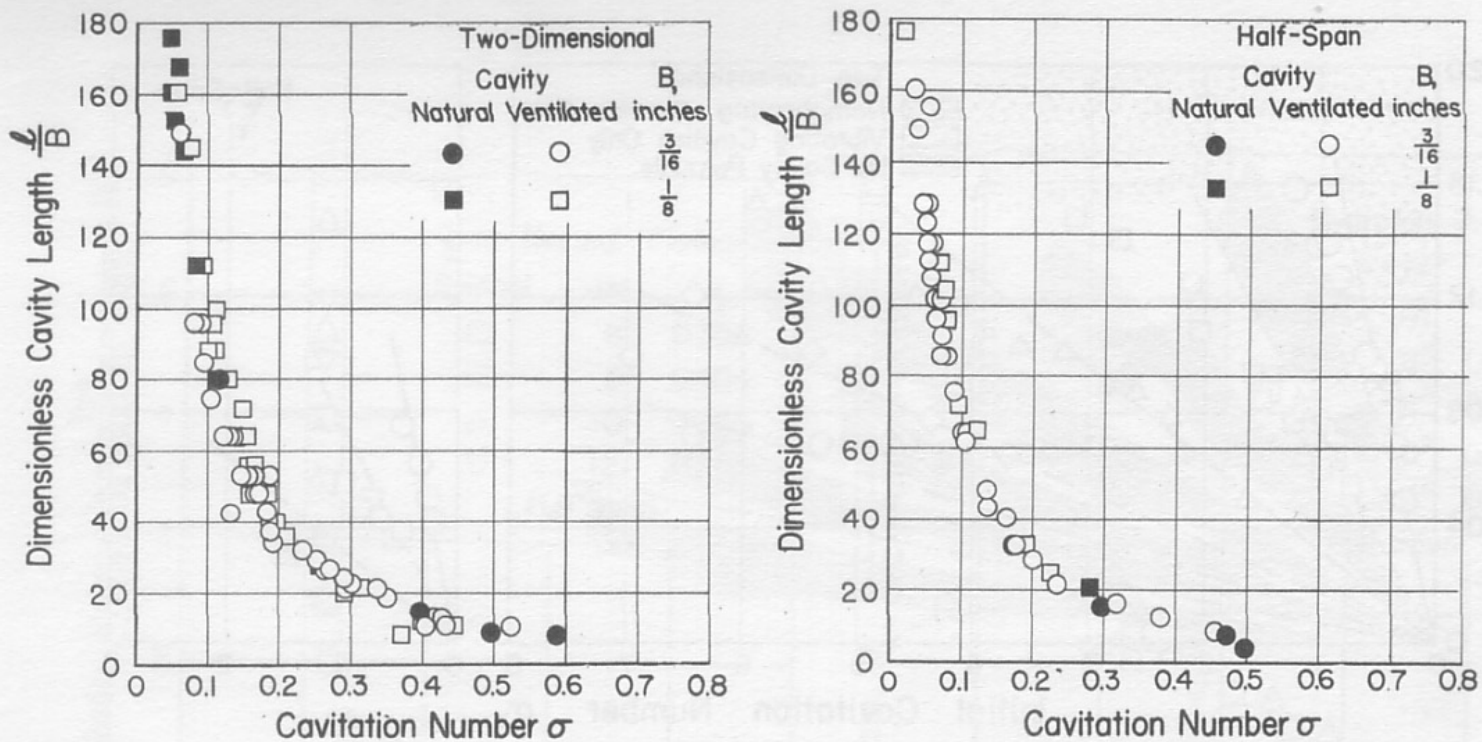

a. Normal Flat Plate
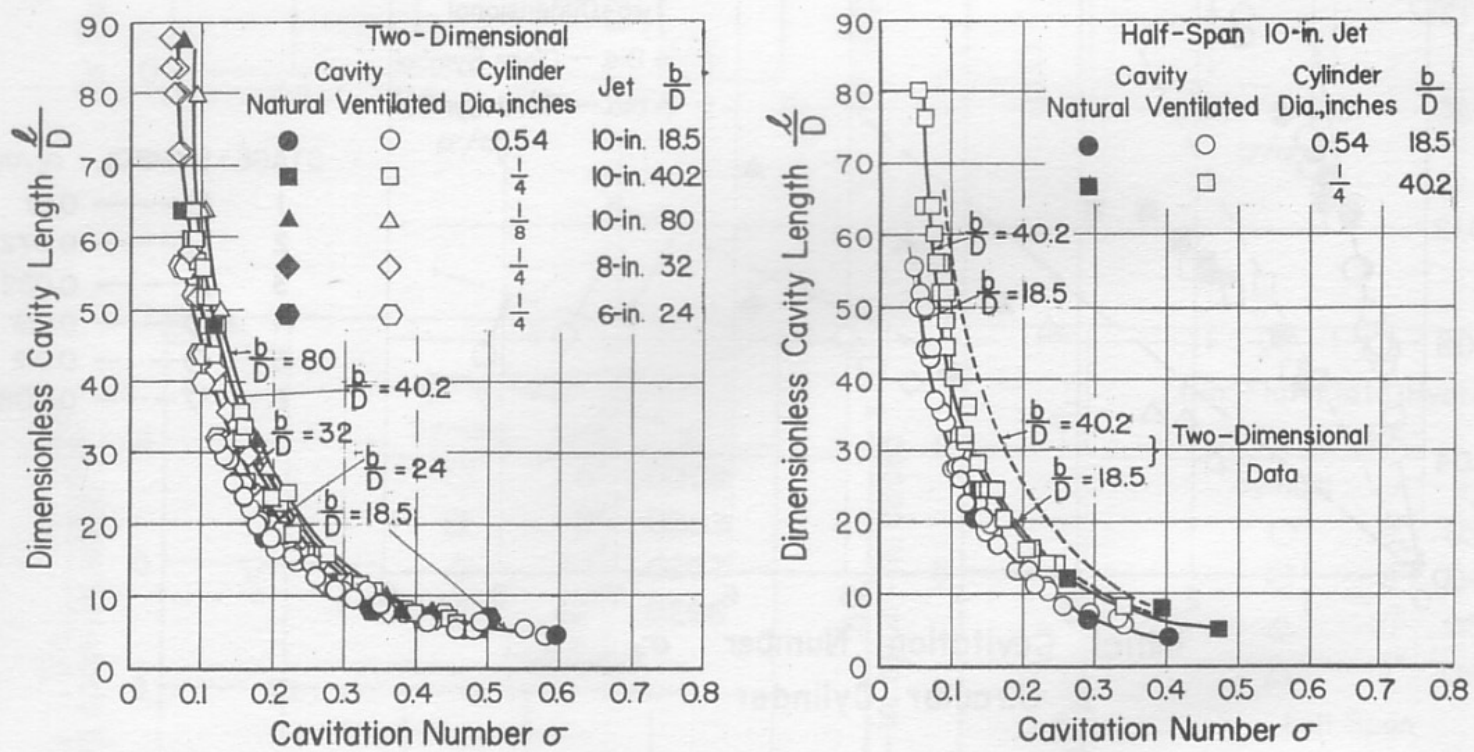

b. Circular Cylinder

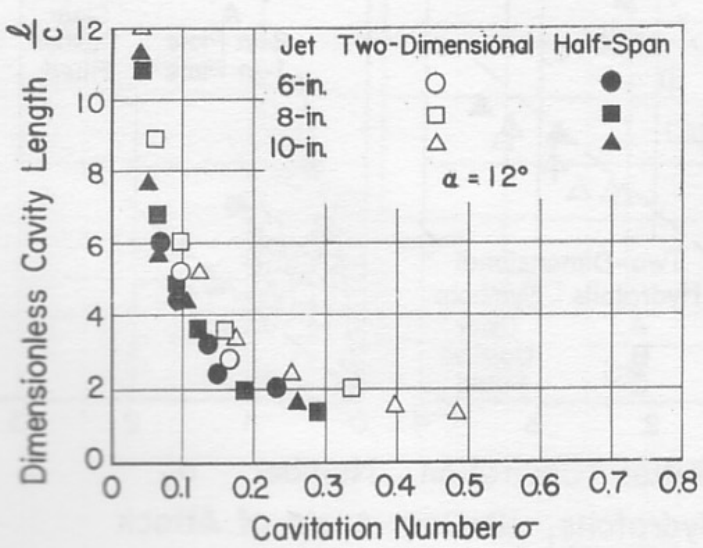

c. Hydrofoil A

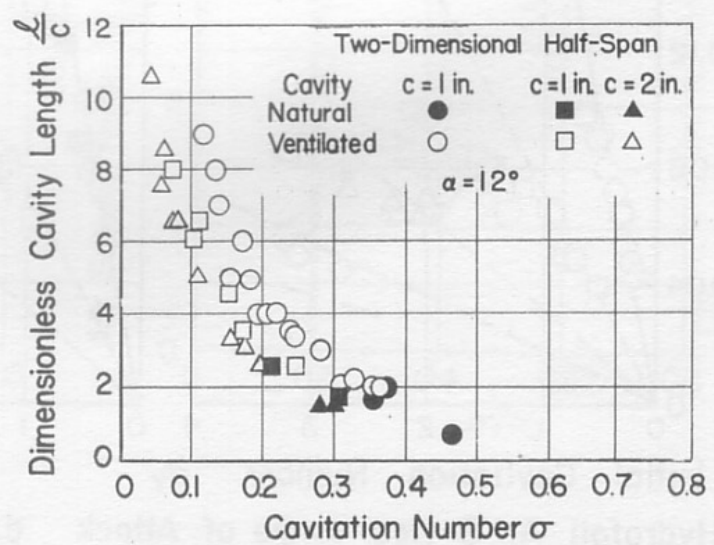

d. Flat Plate Hydrofoil

Fig. 22 - Cavity Length as a Function of Cavitation Number 


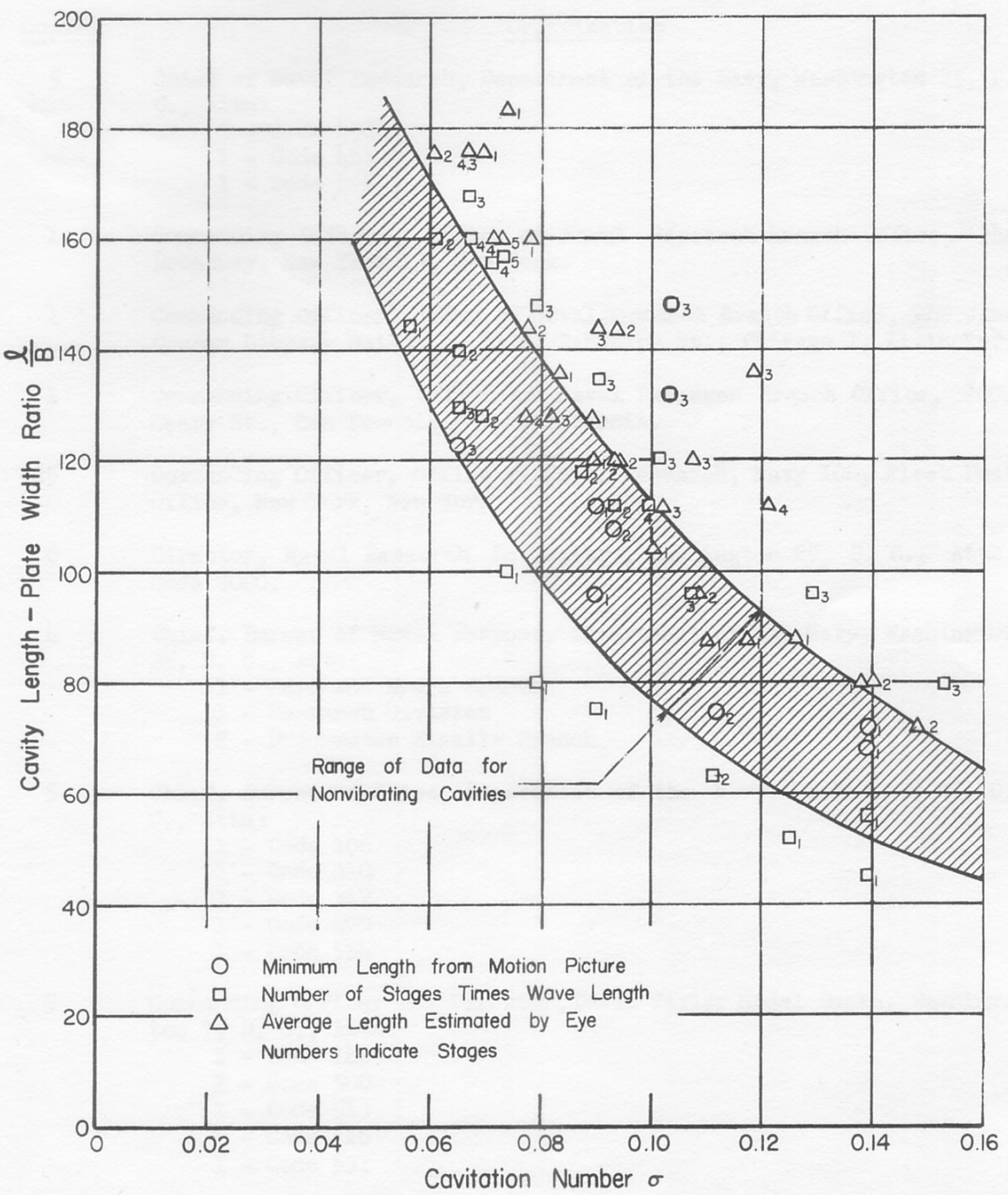

Fig. 23 - Cavity Length for Vibrating Cavities

(Normal plate in 10-in. jet) 
DISTRIBUTION LIST FOR TECHNICAL PAPER NO。 29-B

of the St. Anthony Falls Hydraulic Laboratory

Copies

5

Chief of Naval Research, Department of the Navy, Washington 25, D. C., Attn:

$$
\begin{aligned}
& 3 \text { - Code } 438 \\
& 1 \text { - Code } 463 \\
& 1 \text { - Code } 466
\end{aligned}
$$

1 Commanding Officer, Office of Naval Research Branch Office, 346 Broadway, New York 13, New York.

1 Commanding Officer, Office of Naval Research Branch Office, The John Crerar Library Building, 86 E. Randolph St., Chicago I, Illinois。

1 Commanding Officer, Office of Naval Research Branch Office, 1000 Geary St., San Francisco 9, California.

25 Commanding Officer, Office of Naval Research, Navy 100, Fleet Post Office, New York, New York。

6 Director, Naval Research Laboratory, Washington 25, D。Co, Attn: Code 2000.

4 Chief, Bureau of Naval Weapons, Department of the Navy, Washington 25, D. C., Attn:

1 - Aero and Hydro Branch

1 - Research Division

2 - Underwater Missile Branch

5

Chief, Bureau of Ships, Department of the Navy, Washington 25, D。 C., Attn:

$$
\begin{aligned}
& 1 \text { - Code } 106 \\
& 1 \text { - Code } 310 \\
& 1 \text { - Code } 312 \\
& 1 \text { - Code } 420 \\
& 1 \text { - Code } 554
\end{aligned}
$$

5 Commanding Officer and Director, David Taylor Model Basin, Washington $7, D_{0}$ Co, Attn:

$$
\begin{aligned}
& 1 \text { - Code } 142 \\
& 1 \text { - Code } 500 \\
& 1 \text { - Code } 513 \\
& 1 \text { - Code } 526 \\
& 1 \text { - Code } 591
\end{aligned}
$$

1 Commander, U。 S. Naval Ordnance Test Station, China Lake, California, Attn: Code 753.

1 Officer-in-Charge, Pasadena Annex, U. S。 Naval Ordnance Test Station, 3202 E. Foothill Boulevard, Pasadena, California, Attn: Code P890962。 
$1 \quad$ Commanding Officer and Director, U. S. Naval Engineering Experiment Station, Annapolis, Maryland.

1 Commander, Naval Proving Ground, Dahlgren, Virginia, Attn: Technical Library Division (AAL).

I Commanding Officer, U. S. Naval Underwater Ordnance Station, Newport, Rhode Island, Attn: Research Division.

1 Commander, U. S. Naval Ordnance Laboratory, White Oak, Maryland, Attn: Library Division (Desk HL).

I Mr. W. I. Niedermair, Coordinator of Research, Maritime Administration, 44 G Street, N. W., Washington 25, D. C.

3 National Bureau of Standards, Washington 25, D. C., Attn:

I - Fluid Mechanics Section

I - Dr。 G. B. Schubauer

I - Dr. G. H. Keulegan

1 National Academy of Sciences, National Research Council, 2101 Constitution Avenue, N。Wo, Washington, D. C.

I Superintendent, U. S. Naval Academy, Annapolis, Maryland, Attn: Librarian.

1 Superintendent, U. S. Naval Postgraduate School, Monterey, California, Attn: Librarian.

1 Superintendent, U. S。 Merchant Marine Academy, Kings Point, Long Island, New York, Attn: Captain L。S. McCready, Head, Department of Engineering。

1 Air Force Office of Scientific Research, Mechanics Division, Washington $25, \mathrm{D}, \mathrm{C}$.

I Commanding Officer, Office of Ordnance, Box CM, Duke Station, Durham, North Carolina.

5 Director of Research, National Aeronautics and Space Agency, 1512 H Street, $N_{0}$ Wo, Washington $25, D_{0}$. C

2 Mr。 J. B. Parkinson, Langley Aeronautical Laboratory, National Aeronautics and Space Administration, Langley Field, Virginia.

I Director, Engineering Sciences Division, National Science Foundation, 1520 H Street, N。Wo, Washington, D。C.

10 Document Service Center, Armed Services Technical Information Agency, Arlington Hall Station, Arlington 12, Virginia.

1 Office of Technical Services, Department of Commerce, Washington 25 , D. C. 
California Institute of Technology, Pasadena 4, California, Attn:

1 - Professor M. S. Plesset

1 - Professor T. Y. Wu

1 - Professor A. Acosta

1 - Hydro Lab

University of California, Berkeley 4, California, Attn:

1 - Department of Engineering

1 - Professor H. A. Schade

1 - Professor J. V. Wehausen

1 Director, Scripps Institution of Oceanography, University of California, La Jolla, California.

1 Professor M. Albertson, Department of Civil Engineering, Colorado State University, Fort Collins, Colorado.

IowaInstitute of Hydraulic Research, State University of Iowa, Iowa City, Iowa, Attn:

1 - Professor H. Rouse, Director

1 - Professor L. Landweber

Harvard University, Cambridge 38, Massachusetts, Attn:

1 - Professor G. Birkhoff, Department of Mathematics

1 - Professor G. F. Carrier, Division of Engineering and Applied Physics

Massachusetts Institute of Technology, Cambridge 39, Massachusetts, Attn:

1 - Department of $N_{0} A_{0}$ and $M_{0} E_{0}$

1 - Professor A. T. Ippen, Hydro Laboratory

1 - Iibrary

4 University of Michigan, Ann Arbor, Michigan, Attn:

1 - Professor R。 B. Couch, Department of $N_{0} A_{0}$ and $M_{0} E_{0}$

1 - Professor C.S. Yih, Department of Engineering Mechanics

I - Professor V. Streeter, Department of Civil Engineering

1 - Library

1 Director, St。Anthony Falls Hydraulic Laboratory, University of Min= nesota, Minneapolis 14 , Minnesota。

I Director, Alden Hydraulic Laboratory, Worcester Polytechnic Institute, Worcester, Massachusetts.

1 Director, Ordnance Research Laboratory, Pennsylvania State University, University Park, Pennsylvania.

1 Director, Institute of Mathematical Sciences, New York University, 25 Waverly Place, New York 3, New York。

1 Professor J。J. Foody, Engineering Department, New York State University, Maritime College, Fort Schulyer, New York。 
Technical Library, Webb Institute of Naval Architecture, Crescent Beach Road, Glen Cove, Long Island, New York。

I Professor S. Corrsin, Chairman, Mechanical Engineering Department, The Johns Hopkins University, Baltimore, Maryland.

I Commanding Officer, Office of Naval Research Branch Office, 1030 East Green Street, Pasadena 1, California.

I Director, Woods Hole Oceanographic Institute, Woods Hole, Massachusetts.

1 Society of Naval Architects and Marine Engineers, 74 Trinity Place, New York 6, New York.

1 Engineering Societies Library, 29 W. 39th Street, New York 18, New York.

Stevens Institute of Technology, Davidson Laboratories, 711 Hudson Street, Hoboken, New Jersey, Attn:

$$
\begin{aligned}
& \text { I - Dr。 Jo Breslin } \\
& \text { I - Mr。 Do Savitsky } \\
& \text { I - Library }
\end{aligned}
$$

$1 \quad$ Dr. J. Kotik, Technical Research Group, 17 Union Square West, New York 3, New York。

1 Director, Institute for Fluid Mechanics and Applied Mathematics, University of Maryland, College Park, Maryland。

1 Division of Applied Mathematics, Brown University, Providence 12, Rhode Island。

I Hydrodynamics Laboratory, National Research Council, Ottawa, Canada.

I Professor L。M。Milne-Thomson, Mathematical Research Center, 1118 W. Johnson Center, Madison 6, Wisconsin.

I Dr。 J.M. Robertson, Department of Theoretical and Applied Mechanics, College of Engineering, University of Illinois, Urbana, Illinois.

2 Stanford University, Stanford, California, Attn:

I - Professor J. Ko Venard, Civil Engineering Department

1 - Applied Mathematics and Statistics Laboratory

1 Professor J。B.Herbich, Civil Engineering Department, Lehigh University, Bethlehem, Pennsylvania.

1 Dean J. S. McNown, Department of Engineering Mechanics, University of Kansas, Lawrence, Kansas. 
Professor A. G. Strandhagen, Department of Engineering Mechanics, University of Notre Dame, Notre Dame, Indiana.

2 Polytechnic Institute of Brooklyn, Department of Aeronautical Engineering and Applied Mechanics, 333 Jay Street, Brooklyn I, New York, Attn:

1 - Professor A. Ferri

1 - Professor H. Reissner

1 Professor H. Cohen, IBM Research Center, P. O. Box 218, Yorktown Heights, New York.

1 Professor D. Gilbarg, Applied Mathematics and Statistics Laboratory, Stanford University, Stanford, California.

1 Mir. Leo Geyer, Chief of Preliminary Design, Grumman Aircraft Engineering Corporation, Bethpage, Long Island, New York.

I Mr. W. P. Carl, Jr., Dynamic Developments, Inc., Babylon, Long Island, New York。

1 EDO Corporation, College Point, Long Island, New York。

1 Mr. H. E. Brooke, Hydrodynamics Laboratory, Convair, San Diego 12, California.

I Miami Shipbuilding Corporation, 615 S. W. Second Avenue, Miami 36, Florida.

1 Baker Manufacturing Company, Evansville, Wisconsin.

1 Gibbs and Cox, Inc., 21 West Street, New York 16, New York.

1 Dr. H. Reichardt, Max-Planck-Institut fuer Stroemungsforschung, Goettingem, Boettingerstrass 6/8, West Germany.

1 Director of Research, National Aeronautics and Space Administration, Lewis Research Center, 21000 Brookpark Road, Cleveland 35, Ohio.

Hydronautics, Inc., 200 Monroe Street, Rockville, Maryland, Attn:

1 - Mr. Phillip Eisenberg

1 - Mr. M. P. Tulin

1 Commanding Officer and Director, U. S. Naval Civil Engineering Laboratory, Port Hueneme, California, Attn: Code L54.

1 Micro-Tech Research Company, 629 Massachusetts Avenue, Cambridge, Massachusetts, Attn: Mr. Cohoon. 